\title{
Magnetic field modelling and analysis of uncertainty in
}

\section{archaeological geophysics}

\section{Antonio Schettino ${ }^{1}$, Annalisa Ghezzi ${ }^{1}$, Pietro Paolo Pierantoni ${ }^{1}$}

${ }^{1}$ University of Camerino, School of Science and Technology - Geology Division, Via Gentile III da Varano, 62032 Camerino (MC), Italy

Accepted Manuscript for Publication on Archaeological Prospection

DOI: 10.1002/arp.1729

\section{Corresponding Author:}

Antonio Schettino, University of Camerino, School of Science and Technology, Geology Division,

Via Gentile III da Varano, 62032 Camerino (MC), Italy.

E-mail: antonio.schettino@unicam.it

Tel.: +39-0737-402630 ; +39-0737-402641; Fax: +39-0737-402644 


\title{
Magnetic field modelling and analysis of uncertainty in archaeological geophysics
}

\author{
Antonio Schettino ${ }^{1}$, Annalisa Ghezzi ${ }^{1}$, Pietro Paolo Pierantoni ${ }^{1}$ \\ University of Camerino, School of Science and Technology, Geology Division, Via Gentile III da Varano, 62032 Camerino (MC), Italy
}

\begin{abstract}
One of the major problems in the forward modelling of magnetic anomalies is the assessment of a minimum level of acceptable accuracy in the fit between observed and theoretical anomalies. We present a new approach to the analysis and interpretation of archaeological magnetic anomalies, based on classical algorithms of forward modelling and a new technique of error assessment. This approach allows us to determine geometry, physical properties, and location of buried archaeological features, as well as the occurrence of fires or other historical events that may have affected the observed magnetic signal. Our method starts from the acquisition of total field data, usually in a regular grid arrangement, and proceeds through their reduction to archaeological magnetic anomalies. This reduction is performed subtracting from the observed total field data a polynomial representation of the regional field, on the basis of a rigorous criterion that tries to separate archaelogical anomalies from geological (crustal) contributions. At the next step, a map of the maximum allowed misfit is built, which depends from the estimated uncertainty at each point of the magnetic anomaly field. This map specifies the maximum allowed deviation of theoretical anomalies from the observed values. The last step is the analysis of these anomalies through a new forward modelling tool, with the objective to reconstruct the 3D arrangement of buried features and possibly obtain some information about their history.
\end{abstract}

Keywords: Magnetic anomalies: modelling and interpretation, Magnetic field variations through time, Archaeomagnetism. 


\section{INTRODUCTION}

Although magnetic methods are generally considered among the most important nondestructive techniques in Archaeology, in most cases their application limits to the acquisition of vertical gradient data and the direct interpretation of magnetic lineations in terms of walls or other archaeological features, often without the support of an accurate geophysical analysis. An obvious advantage of this approach is represented by the rapidity and ease with which archaeologists can obtain useful information in view of future excavations. Another advantage is represented by the fact that gradiometers are relatively insensitive to time variations of external disturbing components of the total field, associated with magnetosphere and ionosphere currents, so that the resulting data sets do not require a levelling procedure. In general, gradiometer field intensity decreases with the fourth power of distance, while total field intensity depends from the inverse third power of the distance. Consequently, while gradiometer surveys accentuate the signal associated with shallow features and show greater spatial resolution at low depths, they have decreased sensitivity to deep objects.

Disadvantages in the direct archaeological interpretation of both gradient and total field data include the following issues: 1 . In most cases the location and geometry of a buried artifact are not easily related to size, shape, and position of the corresponding vertical gradient or total field anomalies; 2. Important information about the physical properties of an object, which could have archaeological meaning, is ignored; 3. Information about the burial depth cannot be obtained without a quantitative analysis of the magnetic data; 4 . Nearby objects generate complex anomalies (by the superposition principle) that cannot be interpreted by the simple visual inspection of magnetic maps. However, it is important to stress that vertical gradient anomalies have relatively higher power in the high wavenumbers, so that the corresponding anomalies appear sharper and 
located closer to their causative sources than total field data. Consequently, they are subject to superposition effects to a lesser extent. Finally, Tabbagh (2003) showed that the reduction of anthropogenic disturbances and time variations of the geomagnetic field using appropriate filters gives better results compared to gradiometer measurements.

In this paper, we describe an approach to magnetic prospecting and analysis in Archaeology, which is based on the acquisition of total field data, their reduction to magnetic anomalies, and a computer-assisted modelling of the resulting data set. In this approach, the observed total field data are cleaned by external contributions (solar-quiet diurnal variations) and reduced to magnetic anomalies eliminating the large-scale contribution of Earth's crust and core. It is common practice to perform the latter operation either by least-squares fitting and subtraction of a low-order polynomial surface from the observed data or by suppression of long wavelength components associated with the regional field using an HP filter (Li \& Oldenburg, 1998). In the former approach, the polynomial degree $N$ is often chosen arbitrarily, while the latter method requires anyway some subjectivity in the choice of the cutoff wavelength. In this paper, we will show that $N$ can be chosen on the basis of a rigorous procedure, granted that a gap exists between the range of wavelengths associated with the archaeological anomalies and the regional field bandwidth.

Although both total field and gradient data can be used visually to delineate the geometry of a buried settlement and provide useful information for future excavations, the geophysical perspective may go beyond the simple archaeological interpretation, in so far as we could try to find a physical model of the sources that generated the observed magnetic signal. In this instance, a trial-and-error procedure based on standard forward modelling techniques can be used to generate a magnetization model that explains the observed anomalies. In addition to providing a physical model for the magnetic field observations, the pattern of magnetization could also distinguish between induced 
and NRM components of magnetization, thus giving us the opportunity to detect, in some cases, the occurrence of important historical events. A common issue in the forward modelling procedure is to decide when a satisfactory fit has been obtained between observed anomalies and anomaly values calculated on the basis of the current magnetization model. Although error assessment represents an important aspect of regularized inversion methods (e.g., Zhdanov, 2008 ; Čuma et al., 2012), to our knowledge this problem has never been addressed so far in the forward modelling of potential field data. We will describe a method to solve this problem, based on an estimate of the errors associated with the acquisition and pre-processing of the magnetic data. In this approach, an uncertainty field is built for the survey area, which can be used to define the acceptable local misfit between observed and theoretical anomalies.

\section{ACQUISITION AND PROCESSING OF RAW MAGNETIC DATA}

The typical high-resolution magnetic survey layout is illustrated in Fig. 1A. In this instance, a GPS receiver is used to determine corner location coordinates (and associated confidence regions) of survey areas, whereas the magnetic data are often acquired at $10 \mathrm{~Hz}$ frequency along bidirectional survey lines $\mathbf{L}_{i}$ equally spaced $0.5 \mathrm{~m}$. At a typical operator velocity of $4 \mathrm{~km} / \mathrm{h}$, this sampling frequency translates into an average $11 \mathrm{~cm}$ distance between readings. To assign geographic coordinates to the data set, a computer algorithm calculates the best-fitting rigid transformation from local coordinates to UTM by weighted least squares minimization of corner location errors relative to the measured GPS locations. In the event of rugged terrain, magnetic data acquisition proceeds along curved survey lines (Fig. 1B) and is accompanied by GPS positioning (Schettino et al., 2017). In both cases, a survey is performed only when the value of the solar activity index $K_{p}$ does not indicate magnetic storm conditions $\left(K_{p}<5\right)$. However, even in the case 
of moderately disturbed $\left(K_{p}=4\right)$ or non-disturbed $\left(K_{p}<4\right)$ days, it is good practice to correct the data for the daily variations of the geomagnetic field through a levelling procedure (Luyendyk, 1997) in view of the subsequent phase of modelling. In this instance, in absence of a base station we suggest the following approach. The survey should start with the rapid acquisition along a transverse tie line $\mathbf{T}_{0}$ that crosses the entire survey area (Fig. 1). These data can be considered instantaneous readings at time $t=0$, because $\mathbf{T}_{0}$ is generally travelled in only $1-2 \mathrm{~min}$ in the case of archaeological surveys. Then, the survey is performed normally following the survey lines $\mathbf{L}_{i}$. The geometry illustrated in Fig. 1 implies that the crossover points $\mathbf{C}_{i}$ at the intersections between survey lines and the tie line are travelled twice, so that we have duplicate measurements at these stations. The first reading is a total field intensity, $T\left(\mathbf{C}_{i}, t_{0}\right)$, at position $\mathbf{C}_{i}$ and time $t=t_{0}$ while the second acquisition, $T\left(\mathbf{C}_{i}, t\right)$, represents the field magnitude at the same point and a subsequent time $t$. The crossover error $\varepsilon_{i}(t)=T\left(\mathbf{C}_{i}, t\right)-T\left(\mathbf{C}_{i}, t_{0}\right)$ is the difference between these two readings and does not depend from the crossover point location $\mathbf{C}_{i}$, as it is determined exclusively from the variation of the geomagnetic field at time $t$ relative to the initial time. Therefore, the set of all crossover errors can be used to build a diurnal variations curve $R(t)$ for the geomagnetic field. In other words, the basic idea behind levelling is that the crossover errors, $\varepsilon_{i}$, form a time sequence of data that can be used to estimate the diurnal drift function through a regression procedure. In most cases, the diurnal drift curve $R(t)$ can be obtained from the crossover errors by fitting a cubic polynomial (Fig. 2). This curve is then subtracted from the raw data to remove the external field variations.

Another important step in the standard processing of raw magnetic data consists into the removal of some short-wavelenght artifacts (zig-zags) associated with small errors in the positioning of magnetic readings along the survey lines. To eliminate these artifacts, we apply the following procedure (Fedi \& Florio, 2003 and references therein): 1. High-pass filtering of the raw total field data using a high-order Butterworth filter (e.g., $n=8$ ) and a cutoff frequency depending 
from the corrugation wavelength; 2 . Filtering of the residual grid by a $n$-degree directional cosine in the survey lines direction; 3. Subtraction of the resulting grid from the original raw anomalies.

The first processing step after levelling and decorrugation is the calculation of total magnetic intensity values, cleared by the external variations, on a regular grid arrangement. It should be noted that at the time scale of archaeological prospections (few hours), the cleaned field $T(x, y, z, t)-R(t)$ does not depend anymore from time, because the secular variation of the geomagnetic field ( $~ 80$ nT/yr) gives a negligible contribution even at the scale of few days. During this step, the $z$ coordinate of each observation point and the acquisition time $t$ are stored separately, because they are only important in the subsequent forward modelling phase. In most cases, the procedure that yields the best results is based on a bi-directional gridding algorithm, which enhances transverse (radial) features that extend from line to line perpendicularly to the lines direction. The total field values $T(x, y)$ obtained through this method can be used to calculate magnetic anomalies $\Delta T(x, y)$ by simple subtraction of a reference field intensity $F(x, y)$, so that $\Delta T(x, y)=T(x, y)-F(x, y)$. We shall refer to this grid as the observed anomalies grid. In archaeological geophysics, the reference field $\boldsymbol{F}(x, y)$ should include both the core and crustal (or geological) contributions. We will see that an estimate of its magnitude can be obtained directly from the total field observations. Alternatively, magnetic anomalies can be obtained by application of an HP filter to the grid $T(x, y)$. These anomalies will be called residual anomalies. In principle, the reference field $\boldsymbol{F}(x, y)$ includes only long-wavelength contributes from the Earth's core and crust, thereby in absence of cultural noise the observed anomalies are the expression of a short-wavelength anomalous field that originates from magnetized archaeological features. In this instance, granted that the bandwidth associated with the crustal field does not overlap with the "archaeological" wavelengths, it should be possible to reproduce the grid of observed anomalies by HP filtering of the total field grid $T(x, y)$. However, there is some subjectivity in the choice of the cutoff wavelength for such HP filter. At the same 
time, there is an infinite number of well-behaved functions that potentially can be used to represent the reference field intensity $F(x, y)$.

Classic representations of the reference field magnitude, $F(x, y)$, are based on low-degree polynomial functions (Agocs, 1951 ; Haines, 1968 ; Coles \& Haines, 1979 ; Ardizone \& Herraiz, 2000). In fact, the Earth's magnetic field is harmonic in the region outside the Earth's surface, thereby it has continuous derivatives. Consequently, in any sufficiently small survey area it can be represented by a Taylor's polynomial series with constant coefficients. With this representation, the observed anomalies can be calculated by the following expression:

$$
\Delta T(x, y ; N)=T(x, y)-\sum_{n+m \leq N} a_{n} b_{m} x^{n} y^{m}
$$

where the polynomial coefficients $a_{n}$ and $b_{m}$ are determined by least-squares fitting of the total field values $T(x, y)$. This expression implicitly assumes that the magnetic anomaly field has the same statistical properties of a zero-mean random variable. It also requires a preliminary choice of the polynomial degree $N$ at the right-hand side of (1). For example, we could select a high value of this parameter when the regional field is thought to have short-wavelength components at the scale of the survey area, associated with shallow geological features. Conversely, a low value of $N$ would be used in the case of a regional field dominated by deep sources. However, it is readily understood that such a heuristic approach could not provide correct results in some cases. It is possible to show that the error that arises from an incorrect choice of the polynomial degree may locally reach several tens nT, although the rms error over an entire grid will generally keep below $10 \mathrm{nT}$.

When the reference field is a zero degree polynomial surface, the magnetic anomalies are calculated by subtraction of the core field and a constant crustal contribution. For example, the 
IGRF spherical harmonic representation of the main field includes Legendre polynomials up to degree $n=13$. Therefore, by the Jeans relation (e.g., Schettino, 2014) the minimum wavelength of these polynomials is $\sim 3000 \mathrm{~km}$. Given that the greatest observable wavelength for a squared survey area of size $L$ is $\lambda_{\max }=L$, the core contribution is simply a constant $F(x, y, z)=F_{0}$ at the scale of archaeological survey areas (up to $100 \mathrm{~m}$ ). In this instance, a zero degree polynomial fit of $F$ would practically coincide with the IGRF field intensity up an additive constant that represents the crustal contribution. Therefore, the minimum degree $N$ that can remove a variable crustal field across an archaeological site is one. The problem is, then, how high should be $N$ to provide a correct representation of the core and crustal contributions? In several classic studies the reference field magnitude, $F(x, y)$, was represented by low-degree polynomial functions (Haines, 1968 ; Coles \& Haines, 1979 ; Ardizone \& Herraiz, 2000). Oldham \& Sutherland (1955) proposed a statistical method to constrain the degree $N$, based on a least-squares fitting by orthogonal polynomials. However, in addition to being effective only when the total field grid is sufficiently small, this method requires some amount of subjectivity. Here we propose a different approach, based on repeated comparisons between anomalies calculated through (1), $\Delta T(x, y ; N)$, and residual anomalies, $\Delta T_{r e s}(x, y ; \lambda)$, obtained by HP filtering. The principle at the base of this techinque is very simple and starts from the consideration that for any choice of the polynomial degree $N$, there exists a "bestfitting" cutoff wavelength, $\lambda_{c}$, such that $\Delta T(x, y ; N) \approx \Delta T_{r e s}\left(x, y ; \lambda_{c}\right)$. This is the wavelength that minimizes the rms error, $\varepsilon_{N}(\lambda)$, of the difference grid $\Delta T(x, y ; N)-\Delta T_{\text {res }}(x, y ; \lambda)$. It tells us which wavelengths are removed from the observed total field data $T$ when we subtract the reference field $F$, because a small value of $\varepsilon_{N}(\lambda)$ implies that $\Delta T(x, y ; N)$ can be approximated by the application of an HP filter with cutoff wavelength $\lambda_{c}$. If the crustal field wavelengths have no intersection with the range of wavelengths associated with archaeological features, then there exist two possible trends of the curve $\varepsilon_{N}(\lambda)$ for $\lambda$ in an interval of values that encompasses the archaeological range and the adjacent gap. They are illustrated in Fig. 3. If $\lambda_{c}$ falls in the range of the archaeological anomalies, 
then the curve will show a minimum for $\lambda=\lambda_{c}$ followed by a flat trend when $\lambda$ reaches the gap between crustal and archaeological anomalies. In the case that $\lambda_{c}$ falls in the crustal range, then the curve will not present a minimum at the scale of few hundreds meters, but the fast decay at short wavelengths will be followed anyway by a flat trend across the gap. Clearly, a value of $N$ that generates the latter kind of curve cannot be used to reduce the total field data to anomalies through Expression (1). It is reasonable to expect that in most cases this situation will occurr for $N=0$. Higher values of $N$ are generally associated with cutoff values $\lambda_{c}$ that fall within the range of the archaeological wavelengths, thereby it is quite reasonable to select the polynomial degree associated with the maximum value of $\lambda_{c}$, independently from the value of the misfit $\varepsilon_{N}\left(\lambda_{c}\right)$. With this choice, we reduce to a minimum the risk to remove archaeological information after the application of Expression (1) to the computation of magnetic anomalies.

Fig. 4 shows total magnetic field intensity grids from test areas at four archaeological sites in Italy and Albania, while Fig. 5 illustrates the corresponding plots of $\varepsilon_{N}(\lambda)$ for different degrees $N$ as functions of the cutoff wavelength $\lambda$. These figures show that the smaller the cutoff wavelength, the greater the polynomial degree that provides the minimum rms error, which is quite intuitive. The four archaeological sites are placed in very different geological environments (terraced plain, hill slope, loess plain, and tuff deposits), but the corresponding plots show very similar features that agree with the considerations discussed above. For example, all the curves have an asymptotic value of $\varepsilon_{N}(\lambda)$ for increasing $\lambda$, and there exists a unique critical zone between 80 and $100 \mathrm{~m}$ such that $\varepsilon_{N}(\lambda)$ does not change significantly for higher values of $\lambda$. This observation supports the hypothesis of a gap between the range of wavelengths associated with the archaeological anomalies and the crustal and core contributions. The curves in Fig. 5 suggest that the maximum wavelength of archaeological features is between 80 and $100 \mathrm{~m}$ and that there are no intermediate wavelength components in the observed magnetic field, possibly with the exception of settlements placed on 
rugged terrain (e.g., hill slopes, Fig. 5B). These data unexpectedly show that $N=1$ is the correct choice in three cases out of four, with a best-fitting cutoff wavelength around $50 \mathrm{~m}$.

The final magnetic anomaly map obtained through application of (1) can be used directly for a qualitative archaeological interpretation. Alternatively, it is possible to build a magnetization model that reproduces the observed anomalies at any desired level of accuracy by a forward modelling procedure. In this instance, a preliminary analysis of the data uncertainty is necessary to avoid the search of solutions that locally fit the observations at a level of accuracy exceeding the values of uncertainty.

The uncertainty of magnetic anomaly values that have been obtained by the survey method and processing procedure described above arises from a variety of errors that come into play both during the data acquisition and the subsequent basic processing. For example, we have 1. Errors in the position attributed to total field readings, 2. Errors associated with the variance of the crossover errors about the diurnal drift curve of regression $R(t)$, 3. Errors due to a wrong selection of the gridding algorithm, 4. Errors associated with a wrong choice of the polynomial degree $N$, 5. Errors resulting from the application of the decorrugation filter, 6. Errors associated with knitting of two or more survey rectangles from the same archaeological area. Here we will consider the uncertainty associated with the first class of errors, which probably represents the main contribution to the total uncertainty. A separate paper will describe in detail the remaining classes. Table 1 lists the expected magnitudes of uncertainty for some common acquisition and processing errors, based on an examination of several archaeological surveys.

Figure 6 shows the three independent components of the maximum positioning error in the case of regular mapped surveys (Fig. 1A), not assisted by specific position control hardware (e.g., 
Bruniaux et al., 2018). The component $\varepsilon_{x}$ results from a curved shape of the rope (e.g., during windy days) and/or small oscillations of the sensor about the rope. It is generally of the order of 10 $\mathrm{cm}$. The $\varepsilon_{y}$ component mainly results from variations of velocity during the operator walk along a survey line. Its magnitude can be estimated taking the half-amplitude of zig-zag artifacts in the total field grid. Finally, a small vertical $\varepsilon_{z}$ component is generally associated with irregularities of the terrain and does not exceed $5 \mathrm{~cm}$ in most cases. A vector $\xi=\xi(x, y)$ having random orientation and magnitude $\xi=\left(\varepsilon_{x}^{2}+\varepsilon_{y}^{2}+\varepsilon_{z}^{2}\right)^{1 / 2} / 2^{1 / 3}$ will be referred to as a positioning error vector. Its magnitude tells us the average deviation of the position attributed to a total field value $T(x, y, z)$ from its true location. In the case of GPS-assisted surveys, for example in rugged terrain conditions (Fig. 1B), the magnitude of a positioning error vector is determined by the GPS accuracy during the data acquisition. We estimate the local uncertainty associated with positioning errors by the following expression:

$$
\varepsilon_{P}(x, y)=\max \{\xi \cdot \nabla T \mid\}=\xi G(x, y)
$$

where:

$$
G(x, y)=|\nabla T|=\sqrt{\left(\frac{\partial T}{\partial x}\right)^{2}+\left(\frac{\partial T}{\partial y}\right)^{2}+\left(\frac{\partial T}{\partial z}\right)^{2}}
$$

is the analytic signal grid of the total field $T$. Expression (3) can be easily evaluated generating the horizontal derivative grids directly from the total field $T(x, y)$ and calculating the vertical derivative in the Fourier domain (e.g., Blakely, 1995). To prove Equation (2), it is useful considering the simple one-dimensional case of random positioning errors along the same direction of a survey line, in order to avoid complex vector notation. Let $T_{0}(x)$ be the true field value at position $x$ along the survey line and let $\xi(x)$ be a random displacement function. The observed field 
$T$ results from the replacement of $T_{0}(x)$ by $T_{0}(x+\xi(x))$ at any position $x: T(x)=T_{0}(x+\xi(x))$. We have:

$$
T(x)=T_{0}(x+\xi(x))=T_{0}(x)+T_{0}^{\prime}(x) \xi(x)
$$

Consequently, the positioning error $\delta T$ associated with a small random displacement in the $x$ direction will be given by:

$$
\delta T(x)=T(x)-T_{0}(x)=T_{0}^{\prime}(x) \xi(x)
$$

The derivative at the right hand side of Equation (5) is clearly unknown, but can be always approximated by the horizontal derivative of the observed total field, $T^{\prime}(x)$, over a sufficiently small neighbourhood $I\left(x_{0}\right)=\left[x_{0}-h, x_{0}+h\right]$ of any assigned point $x_{0}$ :

$$
\delta T(x) \cong T^{\prime}(x) \xi(x)
$$

In fact, by Equation (4) for sufficiently small displacements $\xi$, it results:

$$
T^{\prime}(x)=T_{0}^{\prime}(x)\left[1+\xi^{\prime}(x)\right]+T_{0}^{\prime \prime}(x) \xi(x)
$$

Therefore, integrating over $I\left(x_{0}\right)$ we have:

$$
\int_{I\left(x_{0}\right)} T^{\prime}(x) d x=\int_{I\left(x_{0}\right)}\left\{T_{0}^{\prime}(x)\left[1+\xi^{\prime}(x)\right]+T_{0}^{\prime \prime}(x) \xi(x)\right\} d x
$$


Consequently, if $\left\langle T^{\prime}\right\rangle$ and $\left\langle T_{0}^{\prime}\right\rangle$ are the derivative averages of the observed and true fields, respectively, over the neighbourhood $I\left(x_{0}\right)$, then integrating by parts Equation (8) we obtain:

$$
\begin{aligned}
\left\langle T^{\prime}\right\rangle & =\left\langle T_{0}^{\prime}\right\rangle+\frac{1}{2 h} \int_{I\left(x_{0}\right)}\left[T_{0}^{\prime}(x) \xi^{\prime}(x)+T_{0}^{\prime \prime}(x) \xi(x)\right] d x= \\
& =\left\langle T_{0}^{\prime}\right\rangle+\frac{T_{0}^{\prime}\left(x_{0}+h\right) \xi\left(x_{0}+h\right)-T_{0}^{\prime}\left(x_{0}-h\right) \xi\left(x_{0}-h\right)}{2 h}= \\
& =\left\langle T_{0}^{\prime}\right\rangle+\left.\frac{d}{d x} \delta T\right|_{x=x_{0}}
\end{aligned}
$$

Now, it is reasonable to assume that the positioning error varies smoothly over the survey line, so that for a sufficiently small neighbourhood of $x_{0}$ it can be considered constant. Therefore:

$$
\left\langle T^{\prime}\right\rangle \approx\left\langle T_{0}^{\prime}\right\rangle
$$

This result proves that the spatial derivatives of the observed field can be used to estimate the uncertainty due to positioning errors. The one-dimensional version of Equation (2) promptly follows replacing in Equation (6) the average displacement $\xi$.

\section{MODELLING OF ARCHAEOLOGICAL ANOMALIES}

We use a computer-assisted procedure of forward modelling to generate magnetization models that explain the observed anomalies. To this purpose, a dedicated computer program, ArchaeoMag, was developed to operate on UTM georeferenced grids of archaeological anomalies. It can be freely downloaded at: http://www.serg.unicam.it/Downloads.htm. The program assumes that the 
anomalies have been determined through the correct application of Equation (1), or an equivalent method of total field data reduction, and that an uncertainty grid has been generated for these data (Equation 2). ArchaeoMag allows to define three basic classes of shapes and a composite structure, corresponding to common archaeological features: 1. Spheres (magnetic dipoles), 2. Rectangular prisms, 3. Generic vertical prisms, and 4. Stairways (Fig. 7). In addition to have some geometry and location, a magnetic source is described by its minimum and maximum burial depths, a magnetic susceptibility, $\chi$, and a remnant magnetization vector $\boldsymbol{M}_{R}=\left(M_{R}, D_{R}, I_{R}\right), M_{R}, D_{R}$, and $I_{R}$ being the NRM magnetization intensity, declination, and inclination, respectively. The program calculates automatically the induced magnetization vector, $\boldsymbol{M}_{I}$, and the total magnetization vector, $\boldsymbol{M}$, of each body by the following expressions:

$$
\begin{aligned}
& \boldsymbol{M}_{I}=\frac{\chi-\chi_{0}}{\mu_{0}} \boldsymbol{F} \equiv \frac{\Delta \chi}{\mu_{0}} \boldsymbol{F} \\
& \boldsymbol{M}=\boldsymbol{M}_{I}+\boldsymbol{M}_{R}
\end{aligned}
$$

where $\boldsymbol{F}=\left(F, D_{0}, I_{0}\right)$ is the regional geomagnetic field, $\mu_{0}$ is the magnetic permeability in the vacuum: $\mu_{0}=4 \pi \times 10^{-7} \mathrm{H} / \mathrm{m}$, and $\chi_{0}$ is the soil volume susceptibility in SI units. It should be noted that Expression (11) does not consider the variability of $\boldsymbol{F}$ in the survey area, because it is assumed a homogeneous reference field with constant intensity $F$, declination $D_{0}$ and $I_{0}$. In fact, even a strong susceptibility contrast $\Delta \chi=10000$ SI would give $\partial M_{I} / \partial F=\Delta \chi / \mu_{0} \cong 7.96 \times 10^{-6} \mathrm{~A} \mathrm{~m}^{-1} \mathrm{nT}^{-1}$, while it is reasonable to assume that the field direction does not change significantly at the scale on an archaeological site. Consequently, the variations of the regional field across the survey area have a negligible effect on the induced magnetization. 
To calculate the anomalous field generated by a magnetized archaeological feature, we have developed in $\mathrm{C}++$ language an intermediate shell over some low-level Fortran routines listed in Blakely (1995). The anomaly associated with a uniformly magnetized sphere at location $\boldsymbol{r}$ can be calculated projecting the anomalous field $\Delta \boldsymbol{F}(\boldsymbol{r})$ generated by a magnetic dipole onto the reference field axis $\hat{\boldsymbol{F}}$. Using the expressions given in Schettino (2014) and taking into account that the magnetic moment $\boldsymbol{m}$ of a sphere can be easily obtained from the total magnetization vector, $\boldsymbol{m}=$ $\boldsymbol{M} V=(4 / 3) \pi \boldsymbol{M} a^{3}, a$ being the sphere radius, we have:

$$
\Delta T(\boldsymbol{r})=\Delta \boldsymbol{F}(\boldsymbol{r}) \cdot \hat{\boldsymbol{F}} \cong \frac{\mu_{0} a^{3} M}{3 r^{3}}[3(\hat{\boldsymbol{M}} \cdot \hat{\boldsymbol{r}}) \hat{\boldsymbol{r}}-\hat{\boldsymbol{M}}] \cdot \hat{\boldsymbol{F}}
$$

In the case of a rectangular prism, Blakely's (1995) routine is based on a solution proposed by Bhattacharyya (1964) for a rectangular prism oriented parallel to the coordinate axes and that extends from some depth $z_{1}$ to the infinity. To calculate the anomaly of a prism having finite thickness and arbitrary orientation, a coordinate transformation is performed from UTM coordinates to a reference frame where the prism has the required parallel orientation. Then, the routine is called twice, once with $z_{1}$ equal to the top of the prism and once with $z_{1}$ equal to the bottom depth but opposite magnetization, and subtracting the second result from the first one. Bhattacharyya's (1964) formula is simply an application of the following general volume integral over a region $\mathbf{R}$ filled by infinitesimal dipolar sources with magnetic moment $d \boldsymbol{m}=\boldsymbol{M}\left(\boldsymbol{r}^{\prime}\right) d V$ to the specific case of a rectangular prism:

$$
\Delta T(\boldsymbol{r})=-\frac{\mu_{0}}{4 \pi} \hat{F}_{i} \frac{\partial}{\partial x_{i}} \int_{\mathbf{R}} M_{j}\left(\boldsymbol{r}^{\prime}\right) \frac{\partial}{\partial x_{j}^{\prime}} \frac{1}{\left\|\boldsymbol{r}-\boldsymbol{r}^{\prime}\right\|} d V
$$


where we have used an index notation with summation convention. In this expression, the observation point is at $\boldsymbol{r}=\left(x_{1}, x_{2}, x_{3}\right)$ and the integral extends over an arbitrarily magnetized region $\mathbf{R}$, whose points $\boldsymbol{r}^{\prime}$ have coordinates $\left(x_{1}^{\prime}, x_{2}^{\prime}, x_{3}^{\prime}\right)$.

The third class of objects that can be modelled using ArchaeoMag is represented by general vertical prisms. In this instance, calculation of anomalies is based on the fact that a vertical prism is a kind of polyhedron having identical upper and lower polygonal faces and $n$ vertical rectangular faces. If magnetization is uniform, the magnetic anomaly of a general polyhedron can be calculated converting the volume integral (14) into a surface integral, so that the anomalous field generated by the object is equivalent to the field associated with a distribution of magnetic "charge" on the body surface:

$$
\Delta \boldsymbol{F}(\boldsymbol{r})=\frac{\mu_{0}}{4 \pi} \oint_{S(\mathbf{R})} \hat{\boldsymbol{r}} \frac{\boldsymbol{M} \cdot d \boldsymbol{S}}{r^{2}}
$$

where $S(\mathbf{R})$ is the boundary of $\mathbf{R}$ and the origin is assumed to coincide with the observation point. The integration surface $S(\mathbf{R})$ at the right hand side of (15) can be approximated by an appropriate $\mathrm{N}$-facets polyhedron, so that this expression can be evaluated summing the contribution of $N$ polygonal facets. ArchaeoMag uses the method proposed by Bott (1963) to calculate the contribution of any polyhedron facet. If the upper surface of a vertical prism is a polygon with $n$ vertices, the program calls the routine once for this top surface, once for the bottom surface, and $n$ times for the lateral vertical facets.

A magnetization model of a buried archaeological settlement relies on the modelling of the observed magnetic anomalies. In the forward modelling approach, the first step in the analysis of a single anomaly requires a guess about the geometry of the magnetic source on the basis of the 
anomaly shape. The burial depth can be assigned either through archaeological considerations or by quantitative methods. The latter include Euler deconvolution techniques (Reid et al., 1990 ; Desvignes et al., 1999) and radially averaged power spectrum analysis (Spector \& Grant, 1970). In ArchaeoMag, the physical parameters of the source are specified assigning an initial intensity and direction for the NRM vector and a starting value for the magnetic susceptibility. The NRM component of the total magnetizaiton vector is predominant in most of the situations that can be studied by magnetic methods. In general, the observation of anomalies associated with induced magnetization requires one or more among the following conditions: 1. A strong susceptibility contrast with the surrounding soil; 2. A random arrangement of natural remnant magnetization (NRM) components (e.g., a random orientation of magnetite grain spins in a paramagnetic matrix, a random build-up of bricks, etc.); 3. A low Koenigsberger ratio $Q=M_{R} / M_{I}$, and 4 . The absence of nearby objects with a significant NRM component. Examples of archaeological features whose anomalies are dominated by induced magnetization contrasts are: graves, historical iron artifacts (Bevan, 2002), ditches and limestone walls. In contrast, remnant magnetization generally produces much stronger anomalies in materials with high Koenigsberger ratio or, more often, when the archaeological structures are fired materials (e.g., bricks) or materials that have been fired at a later time during historical or natural events. When the modelling of an anomaly requires an NRM component, the declination and inclination of the NRM magnetization vector should be chosen on the basis of the strike of the symmetry axis of the anomaly (Fig. 8) and taking into account of the relative amplitudes of the positive and negative peaks (Fig. 9).

At the next step, forward modelling of a magnetic source requires the creation of several magnetic profiles through the anomaly, having different strike. During this phase, the investigator tries to reduce the error curve of each magnetic profile (observed minus theoretical field values) below the uncertainty band through an interactive trial-and-error procedure that modifies 
repeatedly the magnetization model. At each iteration, the NRM parameters and eventually the depth and size of the object are adjusted to progressively minimize the mismatch between the model and observed anomalies along the profiles. The final result is not necessarily what we could find by direct excavation, because of the intrinsic ambiguity of potential field data. However, the availability of archaeological information can help to constrain the model, thereby allowing a realistic reconstruction of a buried settlement.

\section{A CASE STUDY: THE HADRIANOPOLIS ARCHAEOLOGICAL SITE}

We now describe an application of the techniques discussed above through an interesting case study: the Roman settlement of Hadrianopolis in southern Albania (Fig. 10). We performed magnetic and GPR surveys in the area of Gjirokastër, southern Albania, during five campaigns between 2015 and 2017 (e.g., Schettino et al, 2017). Here we will focus on the data acquired at the archaeological site of Hadrianopolis, while a comprehensive analysis of the results obtained during the five missions will be published separately. Total field magnetic data were collected in quiet day $\left(K_{p}<4\right)$ and flat terrain conditions using a Geometrics G-858 caesium vapor magnetometer and the technique described above. The investigated zone was divided in 48 rectangular areas, which were surveyed in a time interval of two years. Such a long time interval required an evaluation of the effect of the secular variation on the modelling of magnetic anomalies. In fact, we use a constant reference field vector $\boldsymbol{F}$ both in the calculation of induced magnetization vectors (Eq. 11) and model anomalies:

$$
\Delta T_{m o d}(x, y, z)=\hat{\boldsymbol{F}} \cdot \Delta \boldsymbol{F}(x, y, z)
$$


In the case of the Hadrianopolis mission, on July $14^{\text {th }} 2015$ the IGRF field at that location had the components: $D_{0}=3.9388^{\circ}, I_{0}=56.6296^{\circ}, F=46331.9 \mathrm{nT}$, with derivatives: $\partial D_{0} / \partial t=$ $0.1023 \% / \mathrm{yr}, \partial I_{0} / \partial t=0.0064 \% \mathrm{yr}, \partial F / \partial t=34.1 \mathrm{nT} / \mathrm{yr}$. Therefore, using the 2016 parameters in modelling gives a maximum change of $\delta F=34.1 \mathrm{nT}$ in the field intensity, which translates into a maximum error $\delta M_{I}=7.96 \times 10^{-6} \delta F \mathrm{~A} \mathrm{~m}^{-1}=2.71 \times 10^{-4} \mathrm{~A} \mathrm{~m}^{-1}$ for a susceptibility contrast $\Delta \chi=$ 10000 SI, which is clearly a negligible value. We now consider the effect of secular variations on the reference field direction, which is used in the calculation of the model anomalies through (16). The corresponding error on the anomalies can estimated as follows:

$$
\begin{aligned}
\delta \Delta T & =\frac{\partial \Delta T}{\partial D_{0}} d D_{0}+\frac{\partial \Delta T}{\partial I_{0}} d I_{0}= \\
& =\left(-\Delta F_{x} \sin D_{0}+\Delta F_{y} \cos D_{0}\right) \cos I_{0} d D_{0}-\left(\Delta F_{x} \cos D_{0}+\Delta F_{y} \sin D_{0}\right) \sin I_{0} d I_{0}+\Delta F_{z} \cos I_{0} d I_{0}
\end{aligned}
$$

If we insert the field parameters for Hadrianopolis in this expression and consider all the possible directions for the anomalous field $\Delta \boldsymbol{F}$, we obtain a maximum error of $5.61 \%$ on the calculated anomalies, which can be still considered acceptable relatively to the typical uncertainty of magnetic data.

The comparative procedure described in a previous section for the selection of the polynomial degree returned values of $N$ between 0 and 2 for all the 48 areas, thereby the total field data were reduced to magnetic anomalies by subtraction of the corresponding low-degree best-fitting polynomials. The complete magnetic anomaly map of the investigated zone is shown in Fig. 10. The corresponding data can be downloaded as Supporting Information. This map reveals two distinct groups of archaeological anomalies. The northern part shows a characteristic NE-SW and NW-SE pattern of linear structures, which are compatible with a regular urban organization of Roman type. The strong isolated dipolar anomalies that are observed in this sector are most likely 
associated with slag pit furnaces or limekilns, suggesting an area that supported the needs of the local community (Bielenin \& Suliga, 2008). The central part of Fig. 10 shows an irregular pattern of strong-amplitude anomalies. It is likely that the characteristics of this area result from the disruption of the regular urban arrangement of the Roman city and a phase of rebuilding during the Byzantine age. In any case, it cannot be studied by traditional methods of visual interpretation. In fact, the apparent chaotic organization of anomalies having an irregular shape most probably results from superposition and coalescence of smaller anomalies. In this instance, forward modelling provides a formidable tool to separate the sources of the signal and create a realistic picture of the buried archaeological structures.

An analysis of the positioning errors associated with the observed anomaly field resulted into the uncertainty grid shown in Fig. 11, whose corresponding data can also be downloaded as Supporting Information. On the basis of the terrain conditions and an analysis of zig-zags of raw total field grids we obtained, by Equation 2, a magnitude of $0.17 \mathrm{~m}$ for the positioning error vectors. It could be argued that the grid of Fig. 11 does not account for the total uncertainty of the observed data. For example, Fig. 2 shows a relevant variability, of the order of some tens nT, of the crossover errors about the estimated diurnal drift curve. However, most of this variability arises once again from positioning errors of the two readings associated with crossover errors, not from short-period geomagnetic field fluctuations (micropulsations), because variations in the period range between $0.1 \mathrm{~s}$ and $10 \mathrm{~min}$ have amplitudes that rarely exceed $1 \mathrm{nT}$ (e.g., Jacobs, 1970). The variance about the diurnal drift curve is not even associated with heading errors, as the maximum Geometrics G858 magnetometer heading error is $1.5 \mathrm{nT}$. Therefore, we believe that the uncertainty grid of Fig. 11 includes most of the uncertainty associated with the acquisition of magnetic data at this site. 
Now we will describe some key features of the anomaly field illustrated in Fig. 10, resulting from the application of the modelling techniques discussed in the previous section. The magnetization models presented below were built assuming a statistical ensemble of sources with average burial depths determined by the radially averaged power spectrum illustrated in Fig. 12 (Spector \& Grant, 1970). Eventually, the burial depths were slightly modified during the modelling procedure to improve the fit. The plot of Fig. 12 shows the presence of two main contributions to the power spectrum. The average depths to the top of the two source distributions were calculated by the following equation (Spector \& Grant, 1970):

$$
z_{\text {top }}=-\frac{s}{4 \pi}
$$

where $s$ is the slope of a linear tract of the power spectrum function. We obtained $z_{\text {top }}=2.16 \mathrm{~m}$ and $z_{\text {top }}=0.14 \mathrm{~m}$ for the deep and shallow distributions of magnetic sources. An interesting feature of the plot in Fig. 12 is represented by the peak at $k_{0}=34.88 \mathrm{~km}^{-1}$. It indicates that the bottom depth to the deepest sources, $z_{b o t}$, can be calculated (Salem et al., 2000). In this instance, the following expression can be used to evaluate this quantity:

$$
k_{0}=\frac{1}{2 \pi\left(z_{b o t}-z_{t o p}\right)} \ln \frac{z_{b o t}}{z_{t o p}}
$$

A numerical evaluation of Equation (19) provided $z_{b o t} \cong 8.48 \mathrm{~m}$ for the deep seated distribution. The top value, $z_{\text {top }}=2.16 \mathrm{~m}$, is in agreement with the top of archaeological structures of Roman age at nearby excavations in the theatre area of Hadrianopolis (Perna, 2013). 
A first group of anomalies (Area A of Fig. 10) is generated by an apparently chaotic assemblage of small features, some of which can be represented by magnetic dipoles, but also includes a linear E-W chain of five anomalies having symmetry about the vertical axis (see transect A-A' in Fig. 13) and amplitudes up to $80 \mathrm{nT}$. Three of these features are $4 \mathrm{~m}$ large composite anomalies that can be modelled by a strongly magnetized central body (with $M_{R}$ ranging between 4 and $17 \mathrm{~A} / \mathrm{m}$ ) partially surrounded by thin and weakly magnetized walls. The characteristic shape of these anomalies (Fig. 13) arises from the contrast between the strongly negative inclination of $\boldsymbol{M}$ in the central body and the positive inclination of the total magnetization vector along the walls. The composition of these contributions results into a negative (blue) circular anomaly surrounded by a positive (red) ring anomaly. On the contrary, two features show a positive (red) circular anomaly surrounded by a negative (blue) ring. The analysis shows that this shape results from the lack of a central body having a magnetization vector directed upwards. The five anomalies are probably representative of a unique kind of archaeological feature, possibly slag pits of large furnaces associated with some kind of metallurgy (Powell et al., 2002 ; Crew, 2002 ; Abrahamsen et al., 2003 ; Smekalova \& Bevan, 2011).

Area B is another interesting zone of the anomaly map shown in Fig. 10. A model for this area is illustrated in Fig. 14. It shows three orthogonal alignments of anomalies having the same orientation of the Roman structures, thereby they could be of the same age. The anomalies resemble those observed in Area A but are smaller both in size and amplitude (see magnetic profiles in Fig. 14). The width of the corresponding model structures is still larger than the size of classic iron metallurgy slag pits found in central Europe (Smekalova et al., 1993 ; Abrahamsen et al., 2003 ; Žalnierius et al., 2007). Again, the characteristic shape and symmetry of the anomaly (central low surrounded by a positive ring) occurs only where the central slag is still preserved. The number of 
structures found in this area suggests the existence of an important smelting centre of Roman or late Antiquity age.

Area $\mathrm{C}$ shows a very different magnetic pattern (Fig. 15), characterized by the superposition of many small anomalies associated with disrupted structures. The archaeological features that are potentially responsible for this signal have been revealed after a considerable modelling work and could not be found by simple visual inspection of the anomaly field. This area is close to the northern boundary of Hadrianopolis, which did not extended beyond the Drino River, thereby part of this assemblage could include remains of the ancient city walls.

The last sector, Area D, shows a regular pattern of weak anomalies having the alignment of Roman age structures (Fig. 16). An interesting feature of this area is represented by the presence of several deep flat structures having strongly negative inclinations $I=-50^{\circ}$ and a declination between zero and $30^{\circ}$ (Fig. 16, green rectangles). This could suggest a purely induced magnetization vector with negative magnitude associated with a negative susceptibility contrast with respect to the surrounding soil. However, this conclusion would not be correct, because in that case the declination would be close to $180^{\circ}$. At the moment, their presence is required by the very straight boundaries of the positive anomalies associated with buried walls but we have no explanation for the magnetization of these structures that presumably correspond to paving slabs.

\section{DISCUSSION}

The approach presented above allows to create realistic magnetization models of archaeological sites even in the case of complex topography, granted that appropriate acquisition and processing of 
total field data have been performed. In our modelling approach, based on the software tool ArchaeoMag, the observed and model grid anomalies are automatically assigned an orthometric height according to an input digital terrain model for the survey area. Therefore, any object in the model acquires local Cartesian coordinates depending from the burial depth specified at the time of its definition as well as from its UTM coordinates. Thus, it is possible to obtain an automatic terrain correction that accounts for the anomaly field distortion associated with topography (Kangazian et al., 2015). In addition, complex procedures such as those suggested by Khesin et al. (1996) and Eppelbaum et al. (2001) are now unnecessary. As mentioned above, the possibility to model NRM components in addition to induced magnetization is an important aspect of our approach, which could be used, in some circumstances, to estimate the age of firing events and help reconstructions of the historical development of a settlement. In fact, when firing is the only event responsible for the acquisition of NRM and it is possible to establish that the artifact has not been moved since that time, we can compare the model NRM declination and inclination with existing master curves of palaeosecular variations, obtaining an age for the magnetization event (e.g., Oldfield et al., 2003 ; Vigliotti, 2006).

It is important to note that the survey method described above relies on a minimal hardware configuration, represented by a single sensor-console pair. Clearly, more sophisticated hardware would allow a reduction of acquisition errors. For example, a moving cart equipped with a sensor array will reduce significantly the positioning errors, while a position control hardware could even diminish them to a negligible value (Bruniaux et al., 2018). Regarding the uncertainty related to external fields variations, a synchronized base station can be used to measure the diurnal changes. These variations can be subtracted directly by the acquisition stream to obtain time-independent total field data. In absence of such additional hardware, the variance of the estimated diurnal drift curve will introduce some additional uncertainty into the observed magnetic anomalies. In solar 
quiet conditions, a diurnal drift curve has a predictable trend (Campbell et al., 1989) that can be approximated by a cubic polynomial at the scale of 1-2 hours. The maximum uncertainty indicated in Table 1 was estimated assuming very unfavourable conditions like those illustrated in Fig. 2. More typical widths of the prediction interval for the regression curve $R(t)$ do not exceed $3 \mathrm{nT}$. In general, magnetic anomalies have a position-independent background uncertainty $\varepsilon_{0}$ that results from the combination of uncertainties associated with the diurnal drift curve $R(t)$, the statistical fitting of the reference surface $F(x, y)$, and the instrumental noise. In normal mapped survey conditions, such background uncertainty does not exceed 3-4 nT.

The procedure of acquisition of total field magnetic data, their reduction to magnetic anomalies, and the principles of uncertainty assessment described in the previous sections have the important objective of allowing an accurate modelling of the archaeological features that are responsible for the observed magnetic data. However, we could guess whether the theoretical distributions of magnetization obtained by forward modelling techniques provide realistic representations of the buried features or there exist many alternative solutions that can explain the observed magnetic field. In other words, how important is non-uniqueness of magnetization distributions in the context of archaeological research? To respond to this question, it is necessary to consider back the origin of non-uniqueness in magnetic field modelling. In general, there are two sources of ambiguity in the results that can be obtained by inverse or direct modelling. The first source of ambiguity arises from the quite obvious observation that infinitely many models can reproduce the observed signal at a degree of accuracy compatible with the uncertainty distribution. For all these models, the amount of misfit between theoretical and observed anomalies falls below the uncertainty at any point in the survey area. Therefore, they are physically equivalent, although only some of them may have archaeological relevance. The second source of ambiguity is associated with the existence of distributions of magnetization that produce an anomalous field $\Delta \boldsymbol{F}=0$. Such distributions are called 
annihilators (Parker, 1977) and can be included in any solution without affecting the fit between theoretical and observed anomalies. In the case of marine or land studies, annihilators are always layers of constant thickness draped on topography (e.g., Parker \& Huestis, 1974). Therefore, in any practical archaeological situation these distributions of magnetization cannot play a role.

\section{CONCLUSION}

In the previous sections, we have presented a new approach to the acquisition and interpretation of magnetic data in archaeological geophysics, which provides a greater quantity of information and allows an easy integration with other geophysical data. In this approach, total field data are acquired, filtered, and reduced to archaeological anomalies according to specific procedures that eliminate or at least reduce arbitrariness in the choice of processing parameters. Then, interactive forward modelling is used to create and edit magnetization models of buried settlements. An important aspect of this approach is represented by the rigorous procedure of reduction of total field data to archaeological anomalies and by an estimation of the uncertainty that affects the data. These data are then interpreted by a rigorous forward modelling procedure that allows to create a realistic representation of the buried structures. In absence of remnant magnetization, our approach is alternative to 3-D inversion techniques that generate magnetic susceptibility maps from total field data in archaeological geophysics (e.g., Cheyney et al., 2015). 


\section{Acknowledgments}

This work was funded by University of Camerino grants (FAR Schettino and Pierantoni). We are grateful to the students that helped us in surveying the site. We also thank two anonymous referees for their very thorough reviews and their helpful suggestions.

\section{REFERENCES}

Abrahamsen, N., Jacobsen, B.H., Koppelt, U., de Lasson, P., Smekalova, T. \& Voss, O., 2003. Archaeomagnetic investigations of Iron Age slags in Denmark, Archaeological Prospection, 10(2), 91-100.

Agocs, W.B., 1951. Least squares residual anomaly determination, Geophysics, 16(4), 686-696.

Ardizone, J. \& Herraiz, M., 2000. Application of the polynomial adjustment to the aeromagnetic survey of the Spanish Mainland, Earth Planets Space, 52(3), 183-196.

Bevan, B.W., 2002. The magnetic properties of archaeological materials, Geosight Tech. Report No. $5,2^{\text {nd }}$ edition, 13 pp., DOI: 10.13140/RG.2.1.3505.5603.

Bhattacharyya, B. K., 1964. Magnetic anomalies due to prism-shaped bodies with arbitrary polarization, Geophysics, 29(4), 517-531. 
Bielenin, K., \& Suliga, I. , 2008. The ancient slag-pit furnace and the reduction process in the light of a new archeological concept and metallurgical research, Metallurgy and foundry engineering, 34, 53-78.

Blakely, R.J., 1995. Potential Theory in Gravity and Magnetic Applications, 441 pp., Cambridge University Press, Cambridge, UK.

Bott, M.H.P., 1963. Two methods applicable to computers for evaluating magnetic anomalies due to finite three dimensional bodies, Geophysical Prospecting, 11(3), 292-299.

Bruniaux, G., Mathé, V., Lévêque, F., Camus, A. \& Ard, V., 2018. Data processing chain for high spatial resolution magnetic survey: Application on the Neolithic site of le Pontet (Charentemaritime, France), Archaeological Prospection, 25(1), 3-16.

Campbell, W.H., Schiffmacher, E.R. \& Kroehl, H.W., 1989. Global quiet day field variation model WDCA/SQ1, Eos Trans. AGU, 70, 66-74.

Cheyney, S., Fishwick, S., Hill, I.A. \& Linford, N.T., 2015. Successful adaptation of threedimensional inversion methodologies for archaeological-scale, total-field magnetic data sets. Geophys. J. Intern., 202(2), 1271-1288.

Coles, R.L. \& Haines, G.V., 1979. Long-wavelength magnetic anomalies over Canada, using polynomial and upward continuation techniques, J. Geomag. and Geoelectr., 31(5), 545-566. 
Crew, P., 2002. Magnetic mapping and dating of prehistoric and medieval iron-working sites in Northwest Wales, Archaeological Prospection, 9(3), 163-182.

Čuma, M., Wilson, G.A. \& Zhdanov, M.S., 2012. Large-scale 3D inversion of potential field data, Geophysical Prospecting, 60(6), 1186-1199, doi: 10.1111/j.1365-2478.2011.01052.x.

Desvignes, G., Tabbagh, A. \& Benech, C., 1999. The determination of the depth of magnetic anomaly sources, Archaeological Prospection, 6(2), 85-105.

Eppelbaum, L.V., Khesin, B.E. \& Itkis, S.E., 2001. Prompt magnetic investigations of archaeological remains in areas of infrastructure development: Israeli experience. Archaeological prospection, 8(3), 163-185.

Fedi, M., \& Florio, G., 2003. Decorrugation and removal of directional trends of magnetic fields by the wavelet transform: Application to archaeological areas, Geophysical Prospecting, 51(4), 261272.

Haines, G.V., 1968. Polynomial estimation of certain geomagnetic quantities, applied to a survey of Scandinavia, Publications of the Dominion Observatory, 37(4), Dept. of Energy, Mines and Resources, Ottawa, pp. 79-113.

Jacobs, J.A., 1970. Geomagnetic Micropulsations, 179 pp., Springer, Berlin, doi:10.1007/978-3642-86828-3. 
Kangazian, M., Oskooi, B. \& Namaki, L., 2015. Investigation of the topography effect on the shape and polarity of the magnetic anomalies. Bollettino di Geofisica Teorica ed Applicata, 56(1), 43-54.

Khesin, B.E., Alexeyev, V.G. \& Eppelbaum, L., 1996. Interpretation of geophysical fields in complicated environments, Springer Science \& Business Media, 352 pp.

Li, Y. \& Oldenburg, D.W., 1998. Separation of regional and residual magnetic field data, Geophysics, 63(2), 431-439.

Luyendyk, A.P.J., 1997. Processing of airborne magnetic data. AGSO J. Australian Geology and Geophysics, 17(2), 31-38.

Oldfield, F., Asioli, A., Accorsi, C. A., Mercuri, A. M., Juggins, S., Langone, L., Rolph, T. , Trincardi, F. , Wolff, G. , Gibbs, Z., Vigliotti, L. , Frignani, M., van der Post, K. \& Branch, N., 2003. A high resolution late Holocene palaeo environmental record from the central Adriatic Sea, Quaternary Science Reviews, 22(2), 319-342.

Oldham, C.H.G. \& Sutherland, D.B., 1955. Orthogonal polynomials: their use in estimating the regional effect, Geophysics, 20(2), 295-306.

Parker, R.L., 1977. Understanding inverse theory, Annual Review of Earth and Planetary Sciences, 5(1), 35-64.

Parker, R.L. \& Huestis, S.P., 1974. The inversion of magnetic anomalies in the presence of topography, J. Geophys. Res., 79(11), 1587-1593. 
Perna, R., 2013. Hadrianopolis (Sofratikë, Albania): monumental and economic Evolution , in L. Bombardieri, A. D’Agostino, V. Orsi, G. Guarducci, S. Valentini (eds.), Identity and Connectivity, Proceedings of the 16th Symposium on Mediterranean Archaeology, Florence, Italy, 1-3 March 2012 (= BAR Int.Ser., 2581), 935-944.

Powell, A.J., McDonnell, J.G., Batt, C.M. \& Vernon, R.W., 2002. An assessment of the magnetic response of an iron-smelting site, Archaeometry, 44(4), 651-665.

Reid, A.B., Allsop, J.M., Granser, H., Millett, A.T. \& Somerton, I.W., 1990. Magnetic interpretation in three dimensions using Euler deconvolution, Geophysics, 55(1), 80-91.

Salem, A., Ushijima, K., Elsirafi, A. \& Mizunaga, H., 2000. Spectral analysis of aeromagnetic data for geothermal reconnaissance of Quseir area, northern Red Sea, Egypt, Proceedings of the World Geothermal Congress, Kyushu-Tohoku, Japan, May 28-June 10, 1669-1674.

Schettino, A., 2014. Quantitative Plate Tectonics, 403 pp., Springer, Berlin, ISBN 978-3-31909134-1.

Schettino, A., Çondi, D., Perna, R., Pierantoni, P.P. \& Ghezzi, A., 2017. Searching for the Antigonea theatre: A magnetic survey in an ancient Epirus city, Archaeological Prospection, 24, 315, DOI: 10.1002/arp.1549.

Smekalova, T.N. \& Bevan, B., 2011. A magnetic exploration for early iron furnaces at Maglegård, tDAR, doi:10.6067/XCV8DN44RC. 
Smekalova, T., Voss, O. \& Abrahamsen, N., 1993. Magnetic investigation of iron-smelting centres at Snorup, Denmark, Archaeologia Polona, 31, 83-103.

Spector, A. \& Grant, F.S., 1970. Statistical models for interpreting aeromagnetic data, Geophysics, 35(2), 293-302.

Tabbagh, J., 2003. Total field magnetic prospection: are vertical gradiometer measurements preferable to single sensor survey?, Archaeological Prospection, 10(2), 75-81.

Vigliotti, L., 2006. Secular variation record of the Earth's magnetic field in Italy during the Holocene: constraints for the construction of a master curve, Geophysical Journal International, 165(2), 414-429.

Žalnierius, A., Navasaitis, J. \& Balčiūnas, D., 2007. The iron smelting site in virbaliūnai ancient settlement, Archaeologia Baltica, 8, 377-386.

Zhdanov, M.S., 2009. New advances in regularized inversion of gravity and electromagnetic data, Geophysical Prospecting, 57(4), 463-478. 
Table 1. Expected magnitude of uncertainty for some acquisition and processing errors [nT]

\begin{tabular}{ccc}
\hline Source of Uncertainty & Grid Average & Standard Deviation \\
\hline Positioning & $<5$ & $<10$ \\
Gridding & $<1$ & $<15$ \\
Diurnal Drift & 0 & $<6$ \\
Polynomial degree $N$ & 0 & $<10$ \\
Decorrugation & 0 & $<5$ \\
Knitting & & $<5$ locally \\
\hline
\end{tabular}




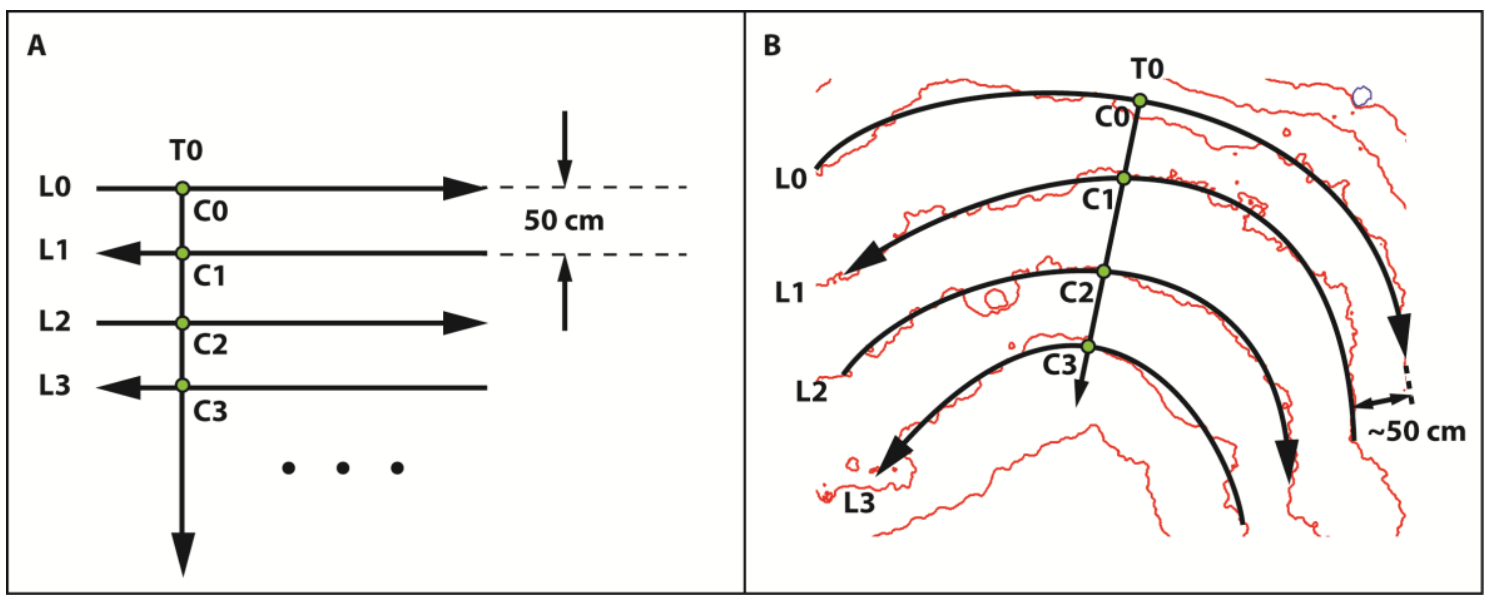

Figure 1.Typical mapped survey layout at sites with planar relief (A) and GPS-assisted survey in presence of complex topography (B). $\mathbf{L} i(i=0,1, \ldots)$ and $\mathbf{T} i$ are respectively survey and tie lines (in black). Red lines are topographic contour lines. $\mathbf{C i}(i=0,1, \ldots)$ are crossover points (green dots) for levelling. 


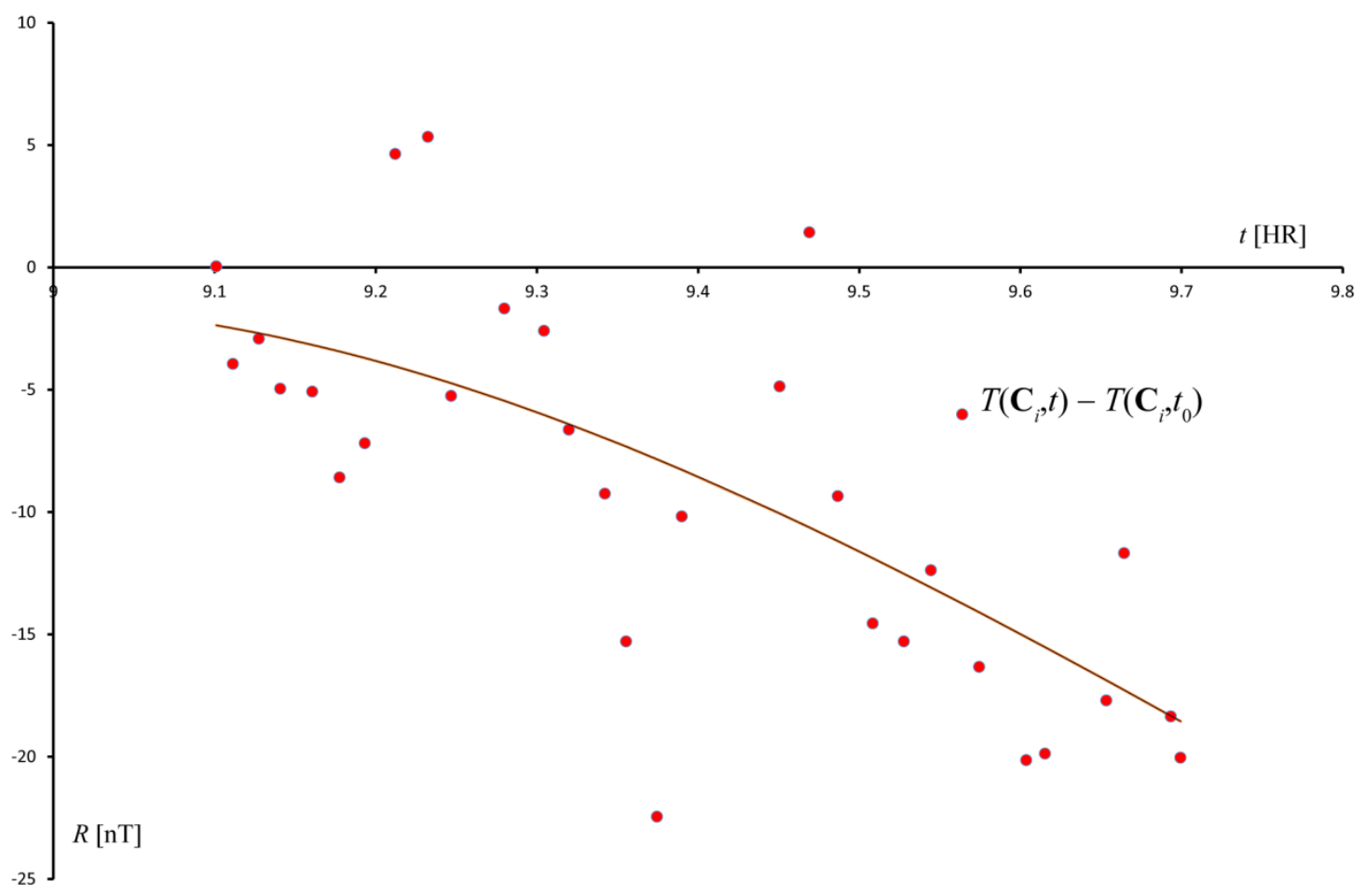

Figure 2. Example of diurnal drift curve $R(t)$, obtained by cubic polynomial fitting of crossover errors $\varepsilon_{i}=T\left(\mathbf{C}_{i}, t\right)-T\left(\mathbf{C}_{i}, t_{0}\right)$ (red dots). These data were acquired at the Antigonea archaeological site (southern Albania) in 2015 (Schettino et al., 2017) in rugged terrain conditions using a GPS. The average positioning error was $\sim 0.5 \mathrm{~m}$, thereby this examples shows very unfavourable survey conditions. 


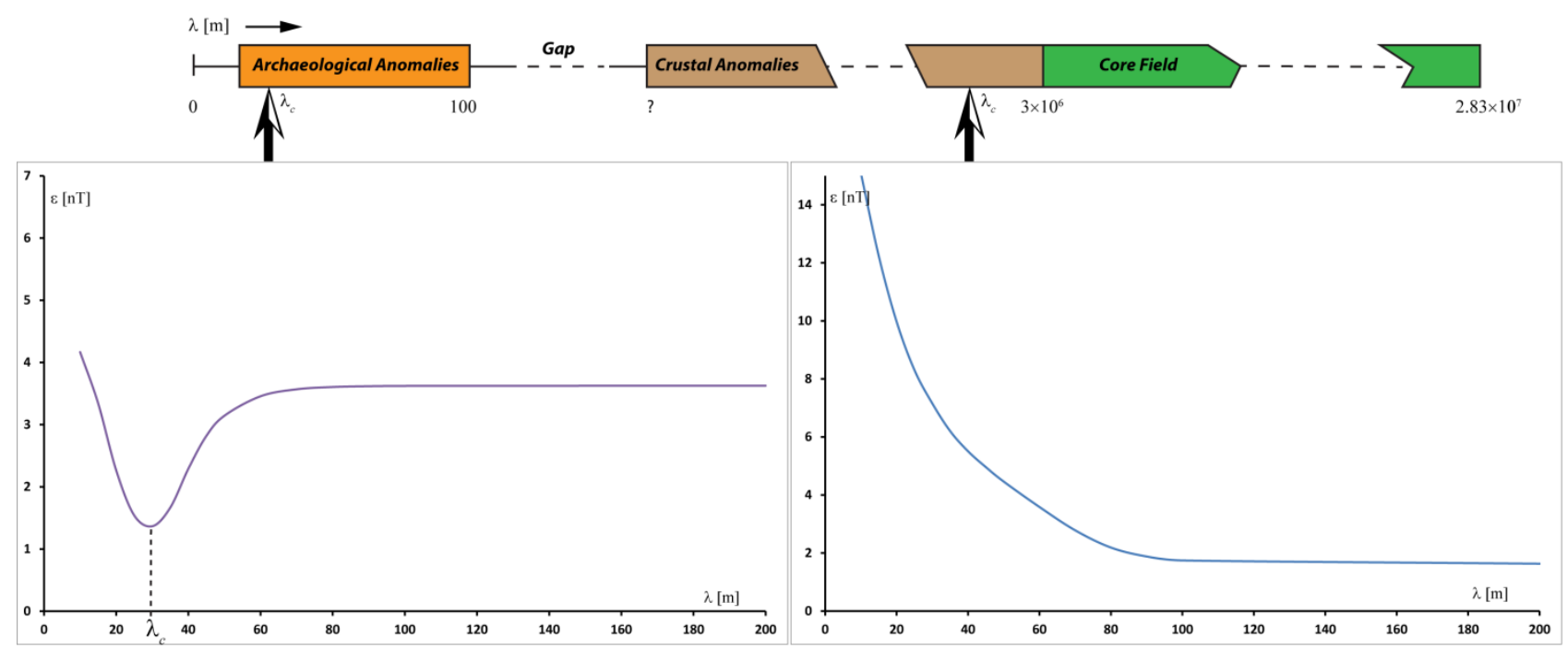

Figure 3. Two different kinds of rms error curves $\varepsilon=\varepsilon(\lambda)$ for the difference grids $\Delta T(x, y ; N)-$ $\Delta T_{\text {res }}(x, y ; \lambda)$ as functions of the cutoff wavelength $\lambda$. When the best-fitting cutoff wavelength $\lambda_{c}$ is within the range of the archaeological anomalies, the curve has a minimum for $\lambda=\lambda_{c}$, followed by an approximately flat trend in correspondence of the gap that separates the crustal anomalies (Left). When the best-fitting cutoff wavelength $\lambda_{c}$ is within the range of the crustal anomalies (Right), there is no minimum at the scale of observation of $200 \mathrm{~m}$, but the curve $\varepsilon(\lambda)$ has anyway a flat trend in correspondence of the gap. 


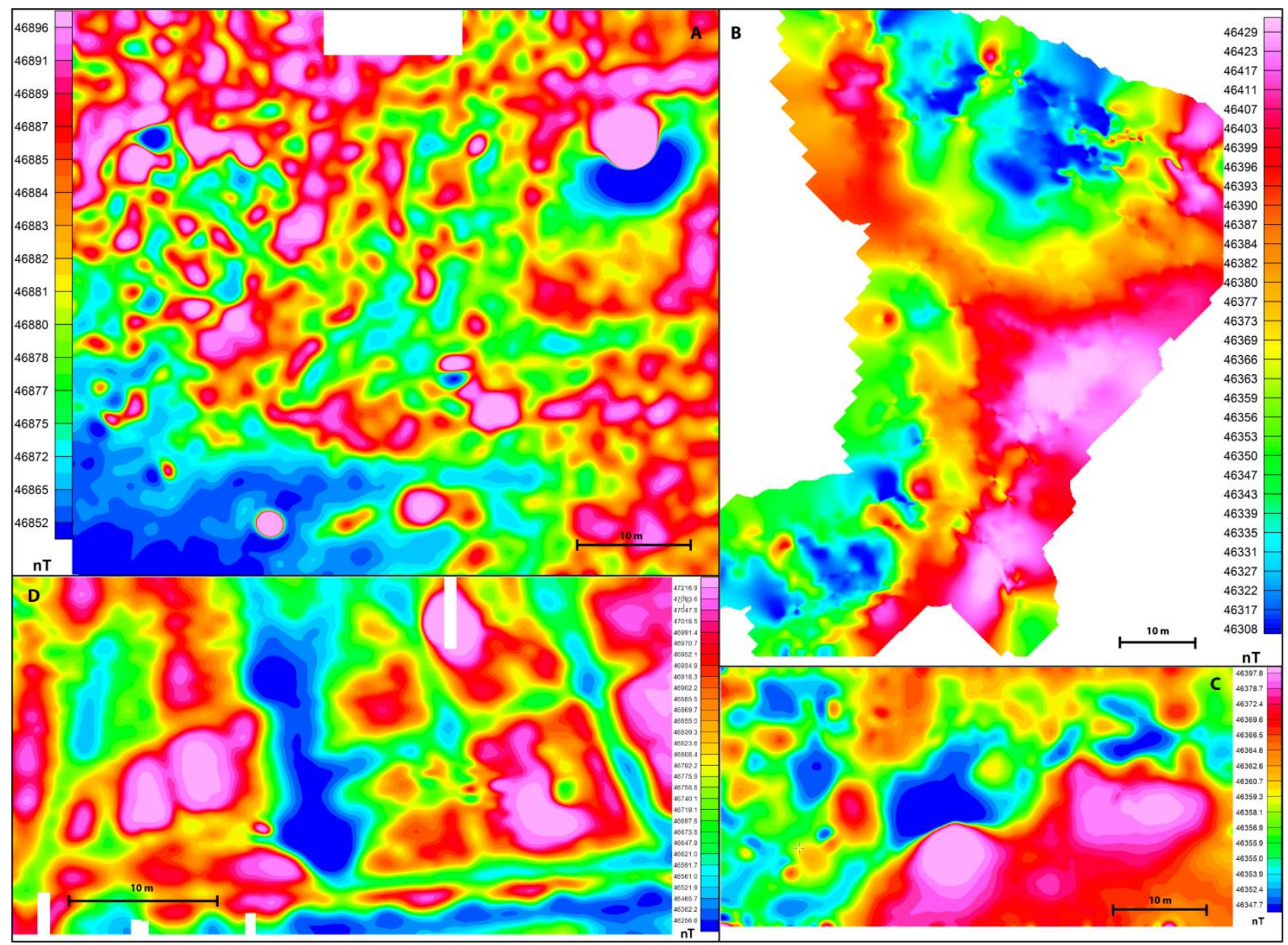

Figure 4. Total field intensity maps at four small survey areas from archaeological sites in Italy and Albania. A: Urbs Salvia, central Italy; B: Antigonea, Albania; C: Hadrianopolis, Albania; D: Hadrian's Villa, near Rome. 


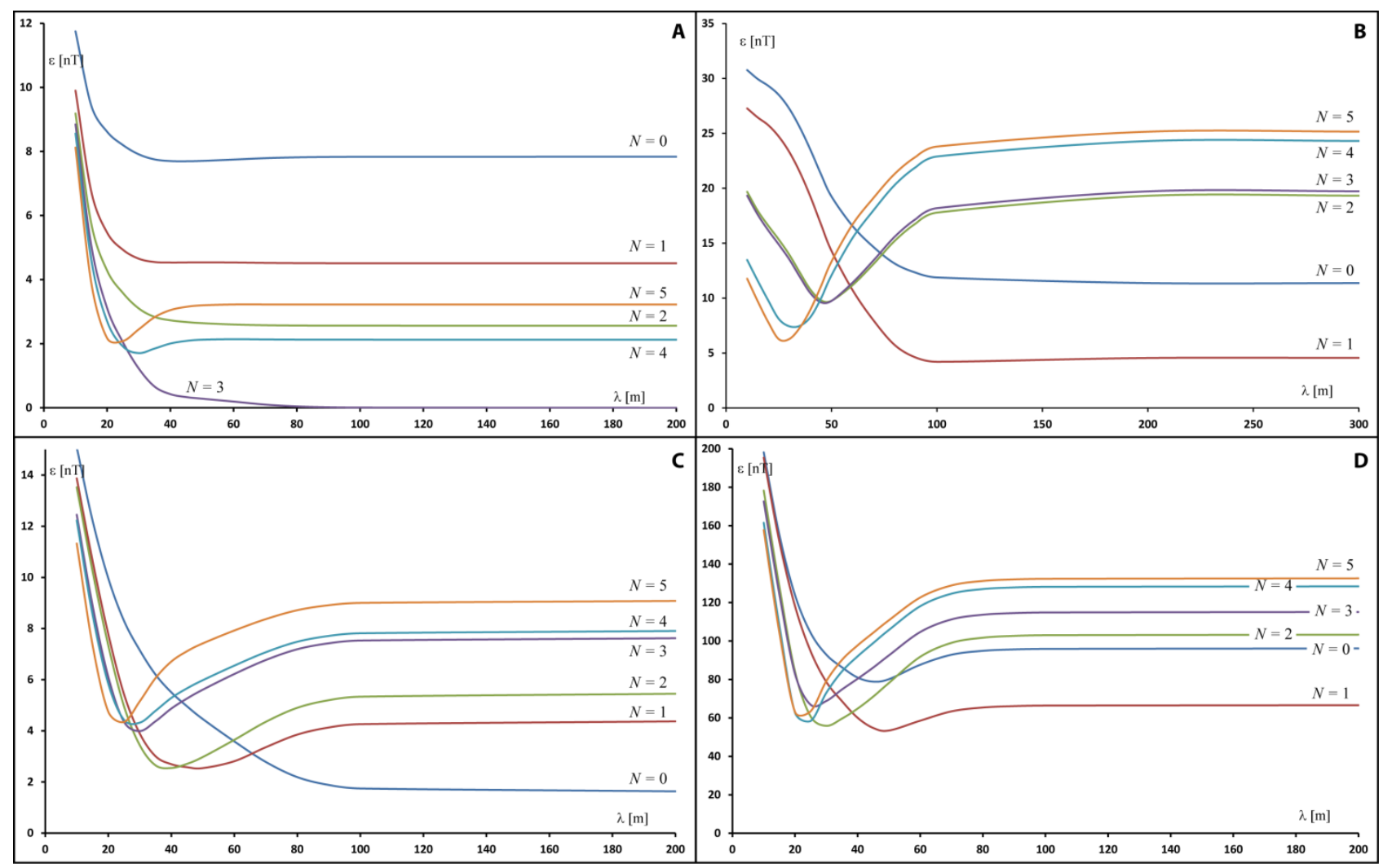

Figure 5. Example curves of rms error $\varepsilon$ of the difference grids $\Delta T(x, y ; N)-\Delta T_{r e s}(x, y ; \lambda)$ for different polynomial degrees $N$ as functions of the cutoff wavelength $\lambda$. A: Urbs Salvia, central Italy; B: Antigonea, Albania; C: Hadrianopolis, Albania; D: Hadrian's Villa, near Rome. The corresponding decorrugated total field measurements are shown in Fig. 4. In all cases, the residual anomalies $\Delta T_{r e s}(x, y ; \lambda)$ were calculated using a high-order Butterworth HP filter $(n=8)$. 


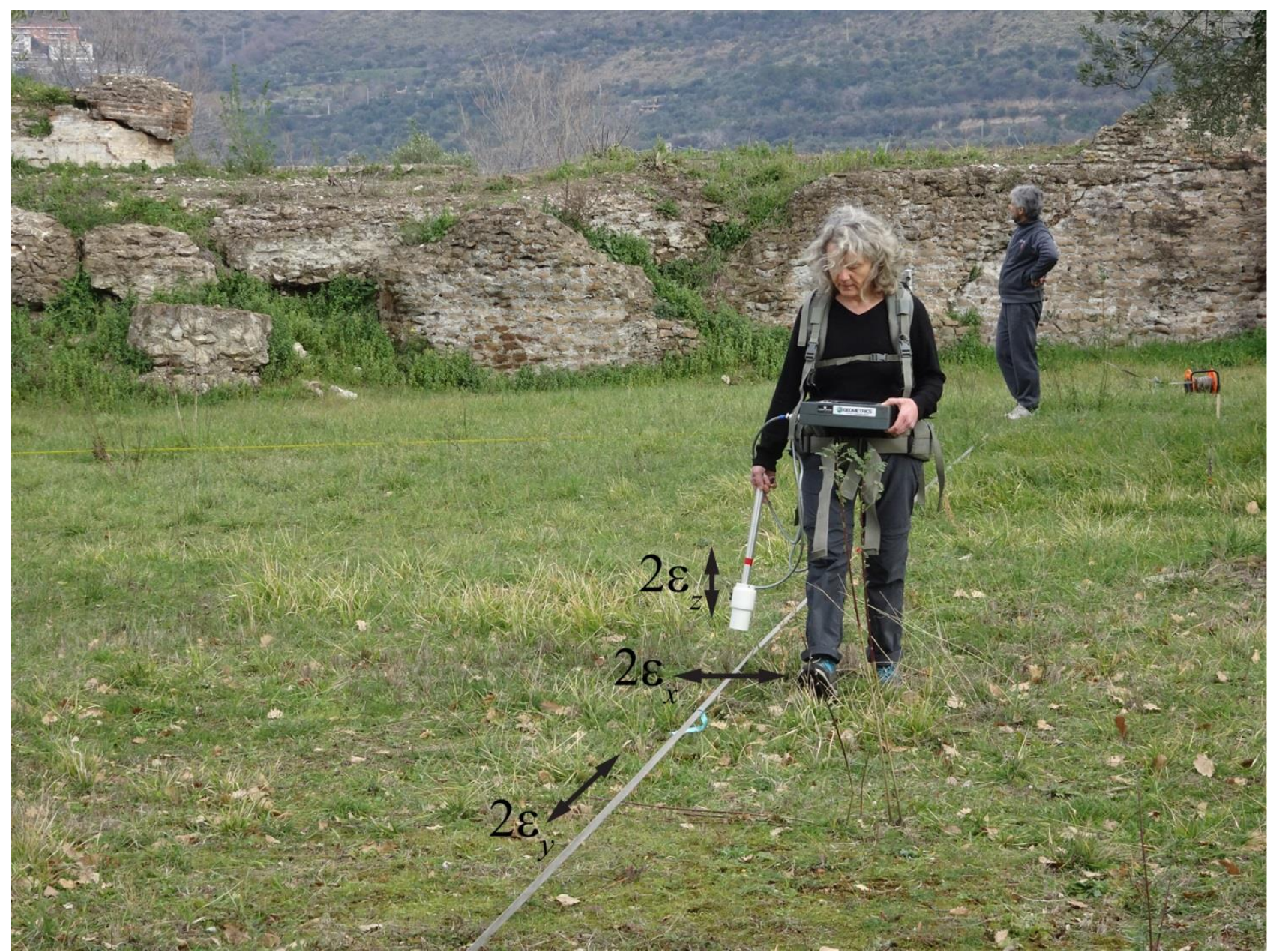

Figure 6. Components of the maximum positioning error vector during data acquisition. 


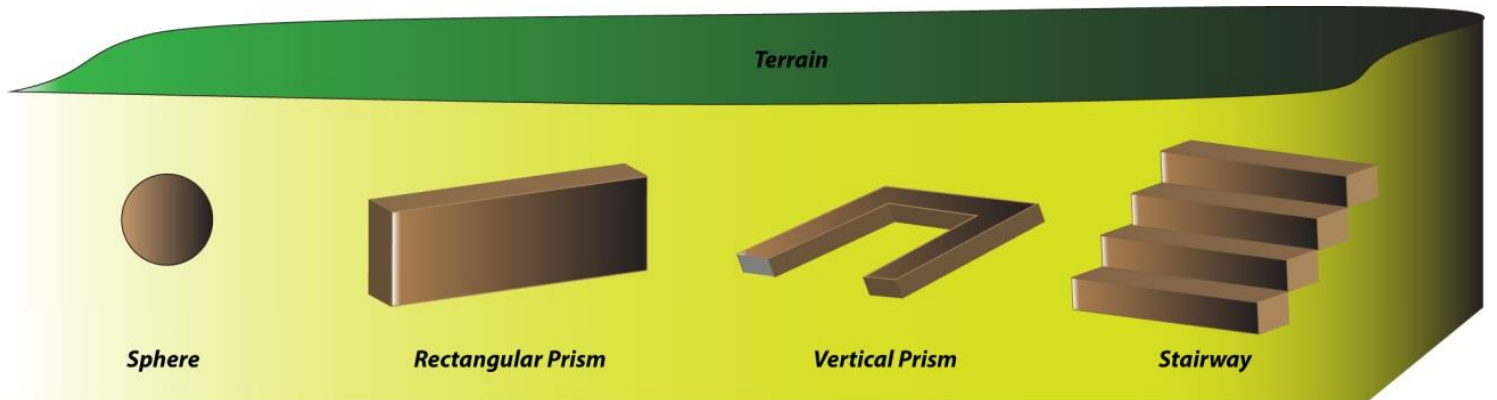

Figure 7. Basic shapes allowed in ArchaeoMag for the definition of magnetized bodies. 

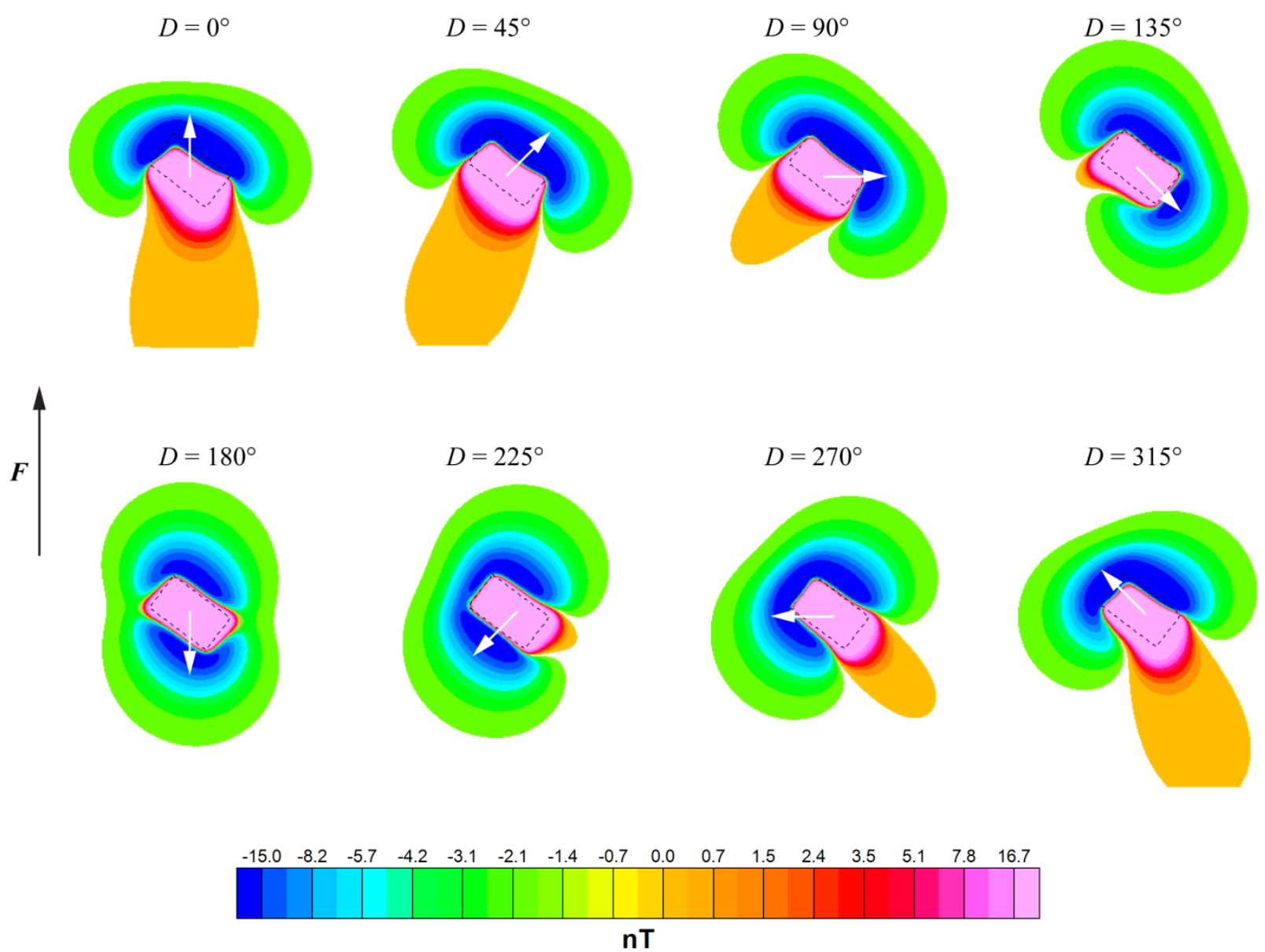

Figure 8. Effect of NRM declination. A NW-SE wall (dashed rectangle) has NRM inclination $I=$ $54^{\circ}$ and $M_{R}=1 \mathrm{~A} / \mathrm{m}$. It is assumed that the ambient field has intensity $F=46824 \mathrm{nT}$, declination $D_{0}$ $=0^{\circ}$, inclination $I_{0}=54^{\circ}$ and that the sensor height is $0.5 \mathrm{~m}$ above a flat terrain. It is also assumed that the susceptibility contrast is zero. The white arrow shows the horizontal projection of the RNM vector. 


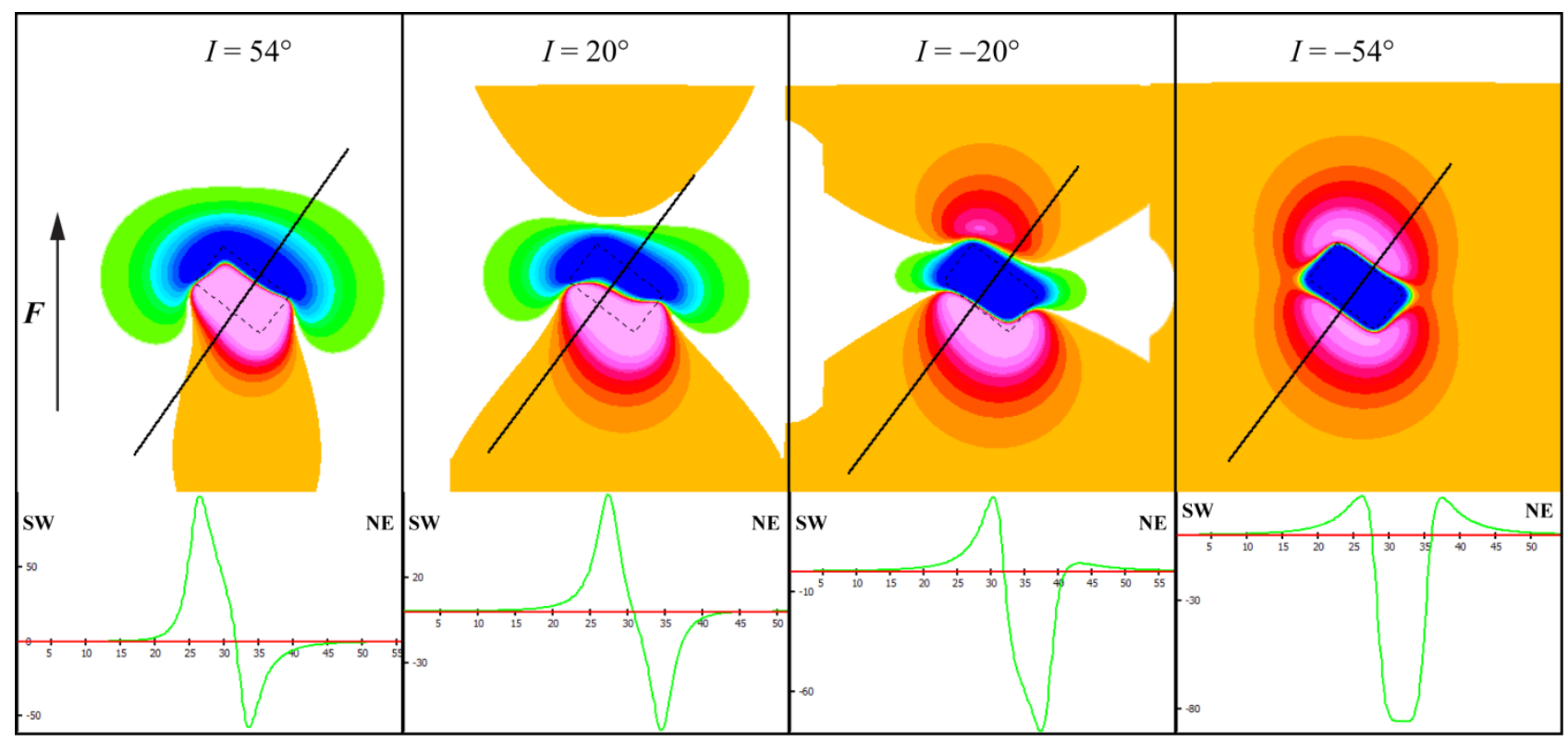

Figure 9. Effect of NRM inclination. A NW-SE wall (dashed rectangle) has declination $D=0^{\circ}$ and $M_{R}=1 \mathrm{~A} / \mathrm{m}$. It is assumed that the ambient field has intensity $F=46824 \mathrm{nT}$, declination $D_{0}=0^{\circ}$, inclination $I_{0}=54^{\circ}$ and that the sensor height is $0.5 \mathrm{~m}$ above a flat terrain. It is also assumed that the susceptibility contrast is zero. The profiles show model anomalies along the traces indicated in the upper panel (black lines). Vertical units are nT, horizontal units are meters. 


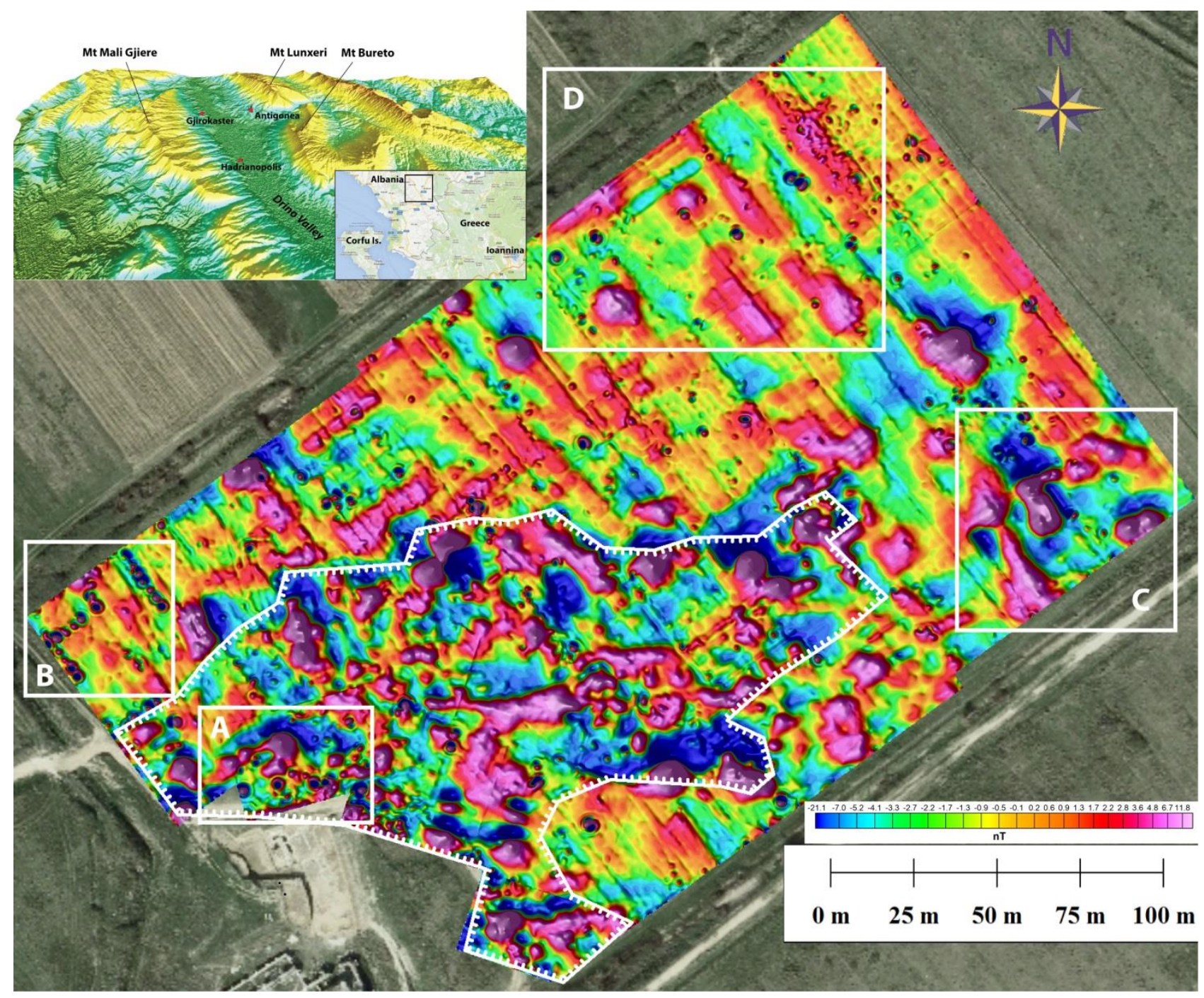

Figure 10. Shaded magnetic anomaly map of the northern sector of the Hadrianopolis settlement. The anomalies reveal the typical urban arrangement of Roman cities, except that in the central part of the map, bounded by the white line with barbs, which shows a chaotical pattern of strongamplitude anomalies, possibly associated with a later Byzantine settlement and some kind of industry. The squares A-D are four representative areas whose anomalies will be studied by forward modelling techniques. 


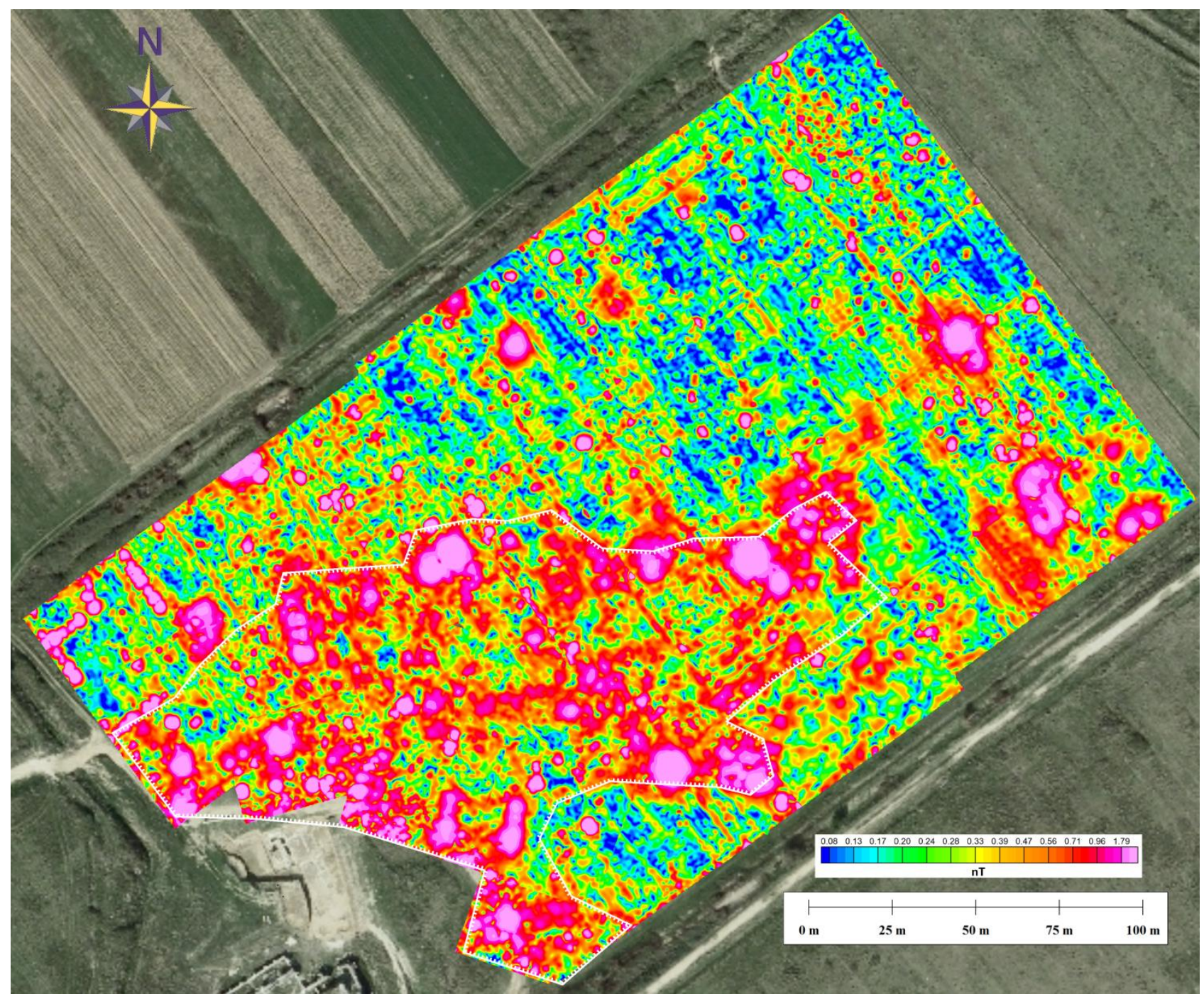

Figure 11. Uncertainty grid for the northern sector of Hadrianopolis. This grid was obtained using (2) and maximum positioning error components $\varepsilon_{x}=0.10 \mathrm{~m}, \varepsilon_{y}=0.18 \mathrm{~m}, \varepsilon_{z}=0.05 \mathrm{~m}$. The $y$ component was estimated on the basis of zig-zag amplitudes of raw total field data. 


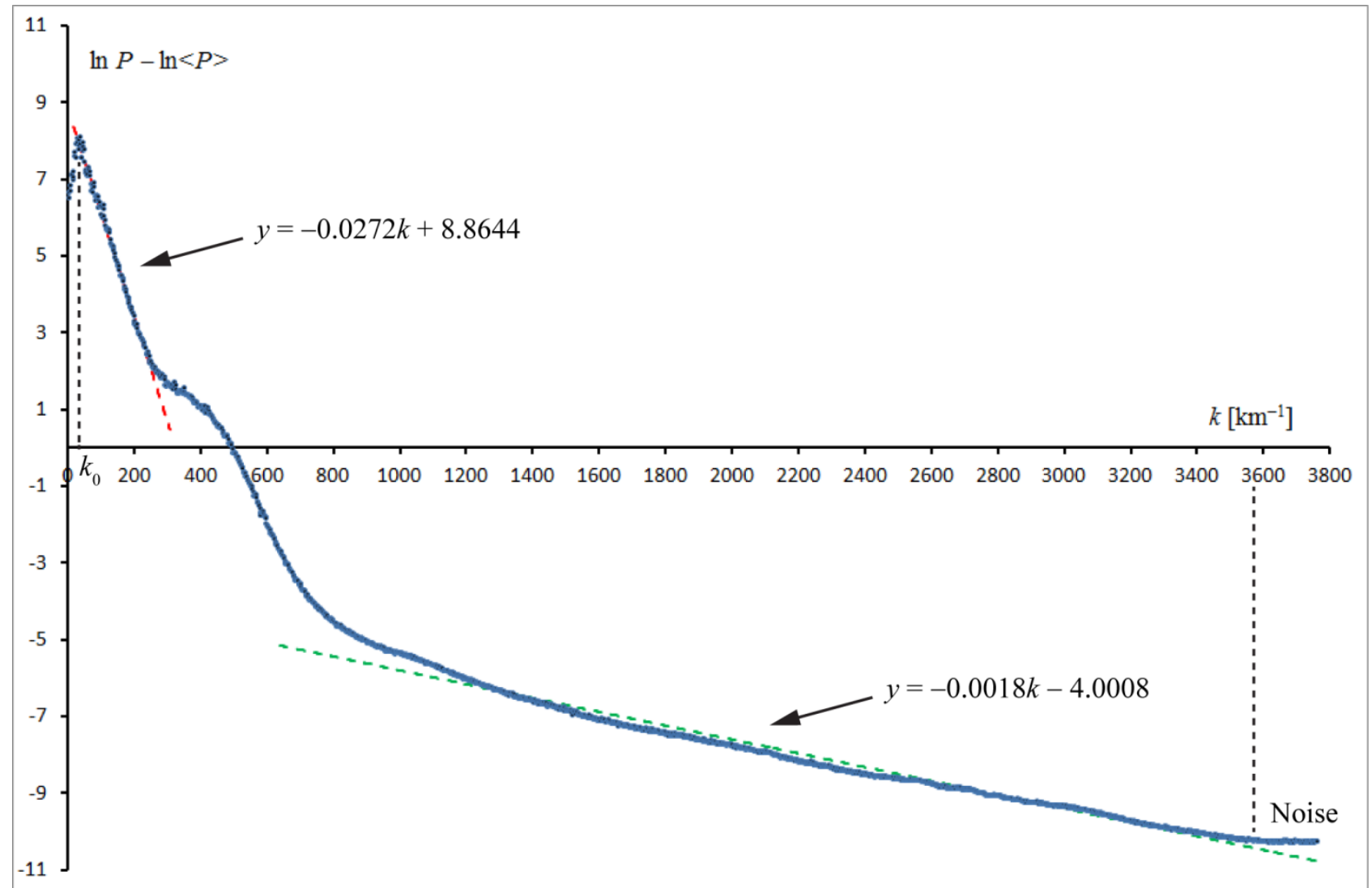

Figure 12. Radially averaged power spectrum of the Hadrianopolis magnetic anomaly grid (see Fig. 10), normalized by subtraction of the log of the average spectrum density. Deep sources are represented by the dashed red fitting line. Shallow sources contribute to the range $950 \leq k \leq 3600$ $\mathrm{km}^{-1}$ (dashed green line). 


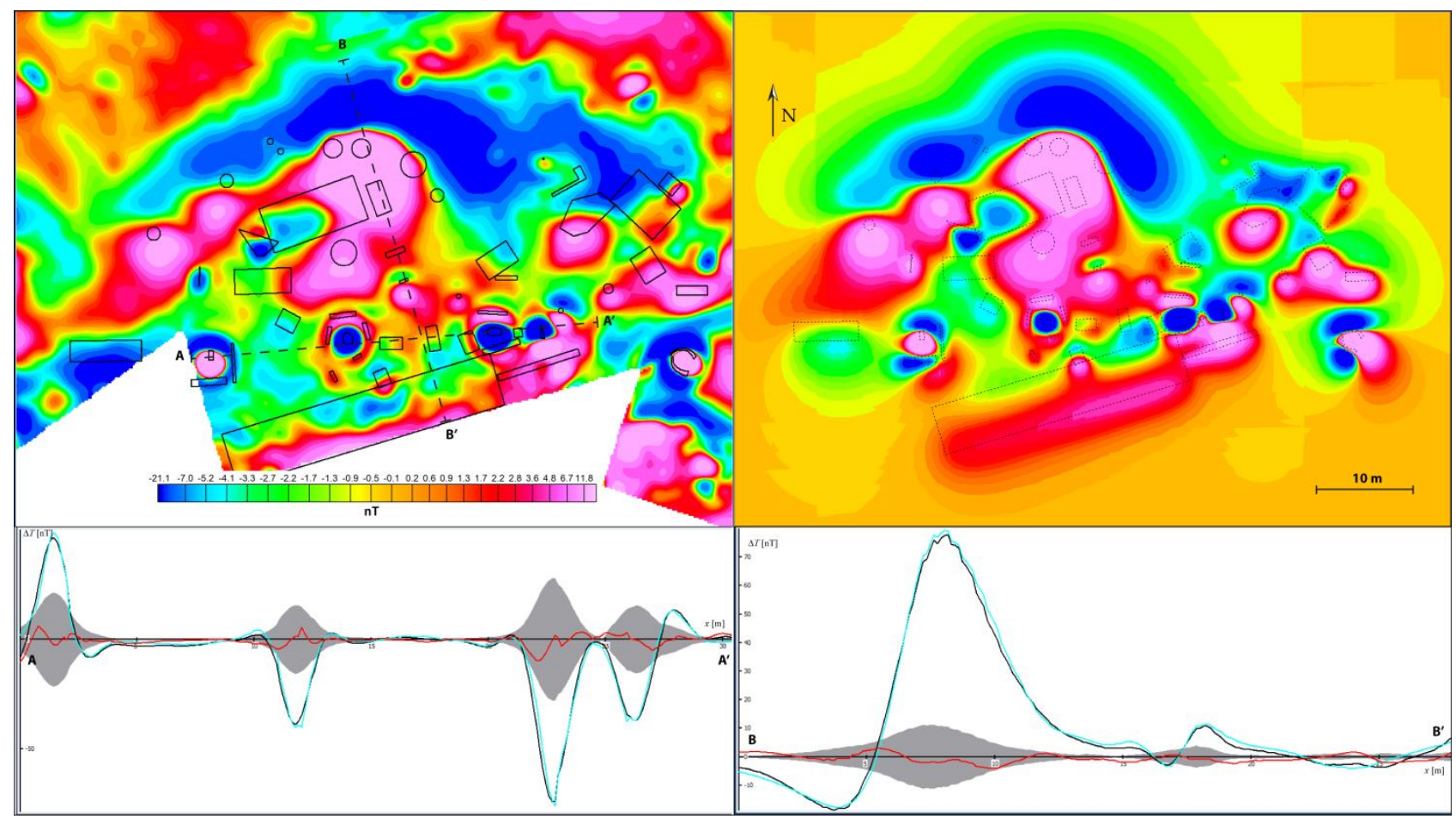

Figure 13. Modelling of area A of the northern sector of the Hadrianopolis settlement (see Fig. 10). Upper left panel shows the observed anomalies and a model of the underground that explains most of the observed signal in this rectangle (black lines). $\mathrm{A}-\mathrm{A}^{\prime}$ and $\mathrm{B}-\mathrm{B}^{\prime}$ are the traces of the two profiles shown in the lower part of the Figure. Upper right panel shows a map of the theoretical anomalies, calculated from the block model. The lower panels illustrate the fit between observed and calculated anomalies along two profiles. The green and black lines show model and observed anomalies, respectively. The red line shows the error curve, obtained subtracting calculated anomalies from the corresponding observed values. The grey areas represent the estimated uncertainty $\varepsilon_{P}$ about the observed curve. The interactive procedure of modelling should bring the red line within this region. 


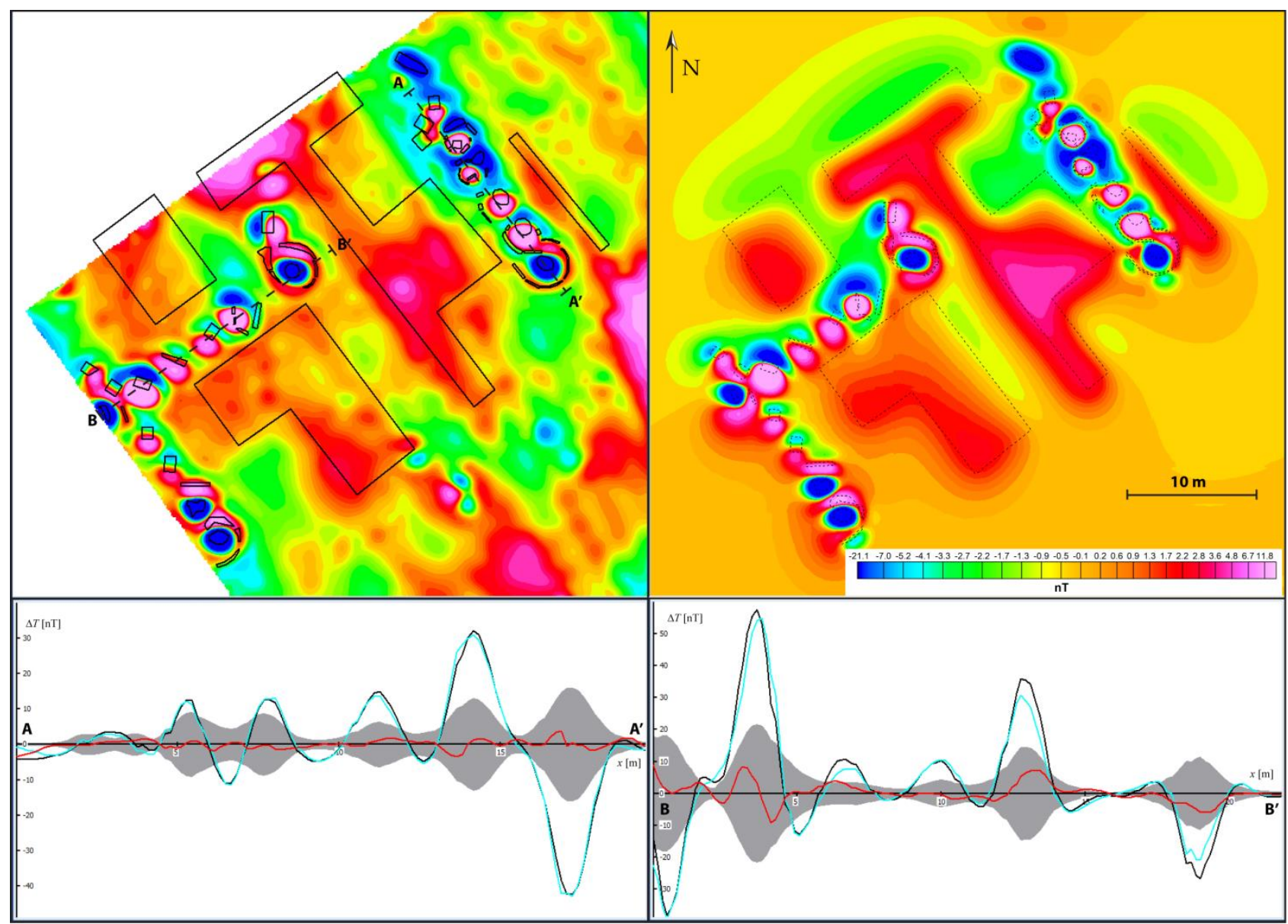

Figure 14. Modelling of area B of the northern sector of the Hadrianopolis settlement (see Fig. 10). Upper left panel shows the observed anomalies and a model of the underground that explains most of the observed signal in this rectangle (black lines). $\mathrm{A}-\mathrm{A}^{\prime}$ and $\mathrm{B}-\mathrm{B}^{\prime}$ are the traces of the two profiles shown in the upper left panels. Upper right panel shows a map of the theoretical anomalies, calculated from the block model. The lower panels illustrate the fit between observed and calculated anomalies along two profiles. 


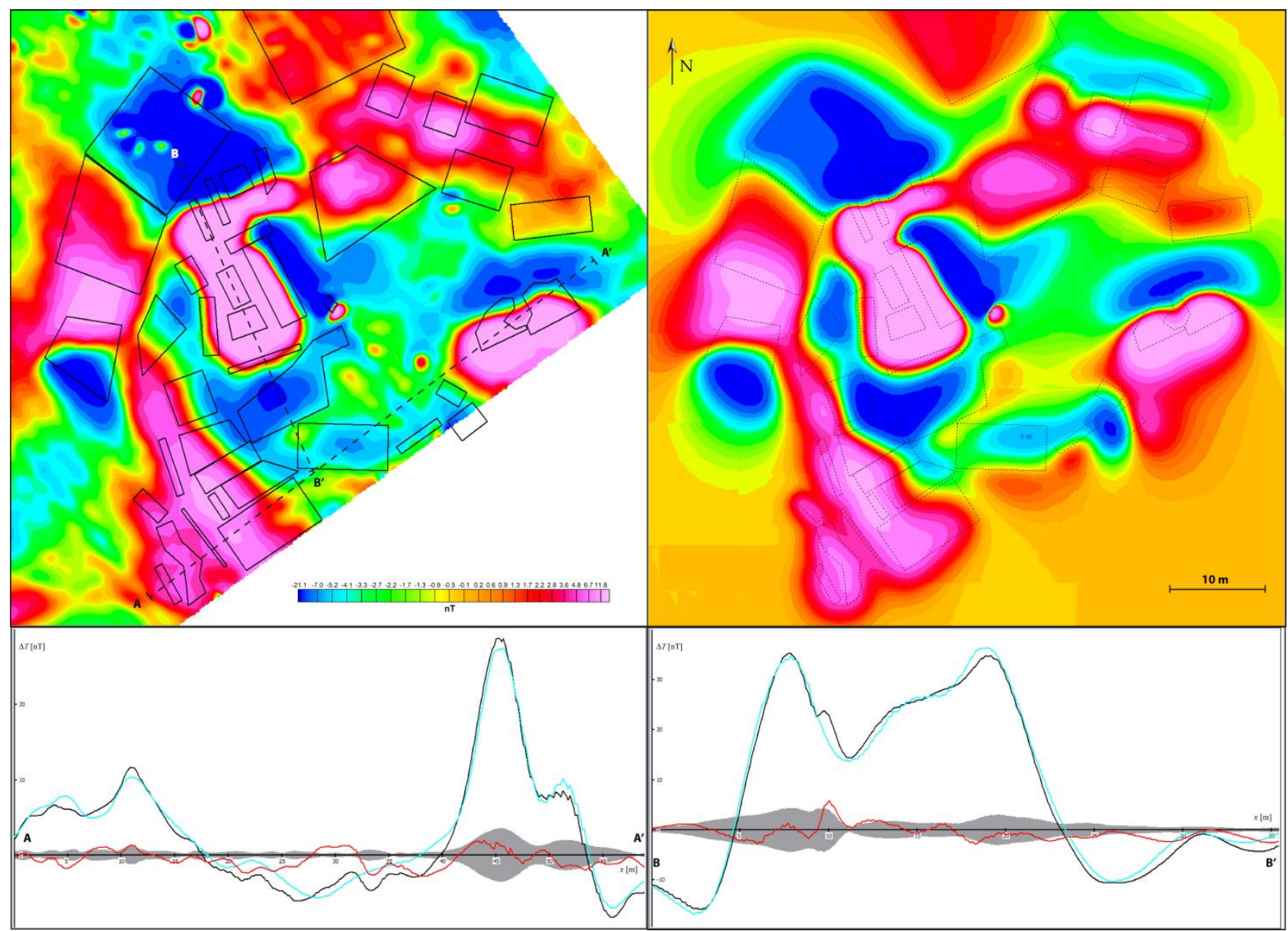

Figure 15. Modelling of area $\mathrm{C}$ of the northern sector of the Hadrianopolis settlement (see Fig. 10). Upper left panel shows the observed anomalies and a model of the underground that explains most of the observed signal in this rectangle (black lines). $\mathrm{A}-\mathrm{A}^{\prime}$ and $\mathrm{B}-\mathrm{B}^{\prime}$ are the traces of the two profiles shown in the upper left panels. Upper right panel shows a map of the theoretical anomalies, calculated from the block model. The lower panels illustrate the fit between observed and calculated anomalies along two profiles. 


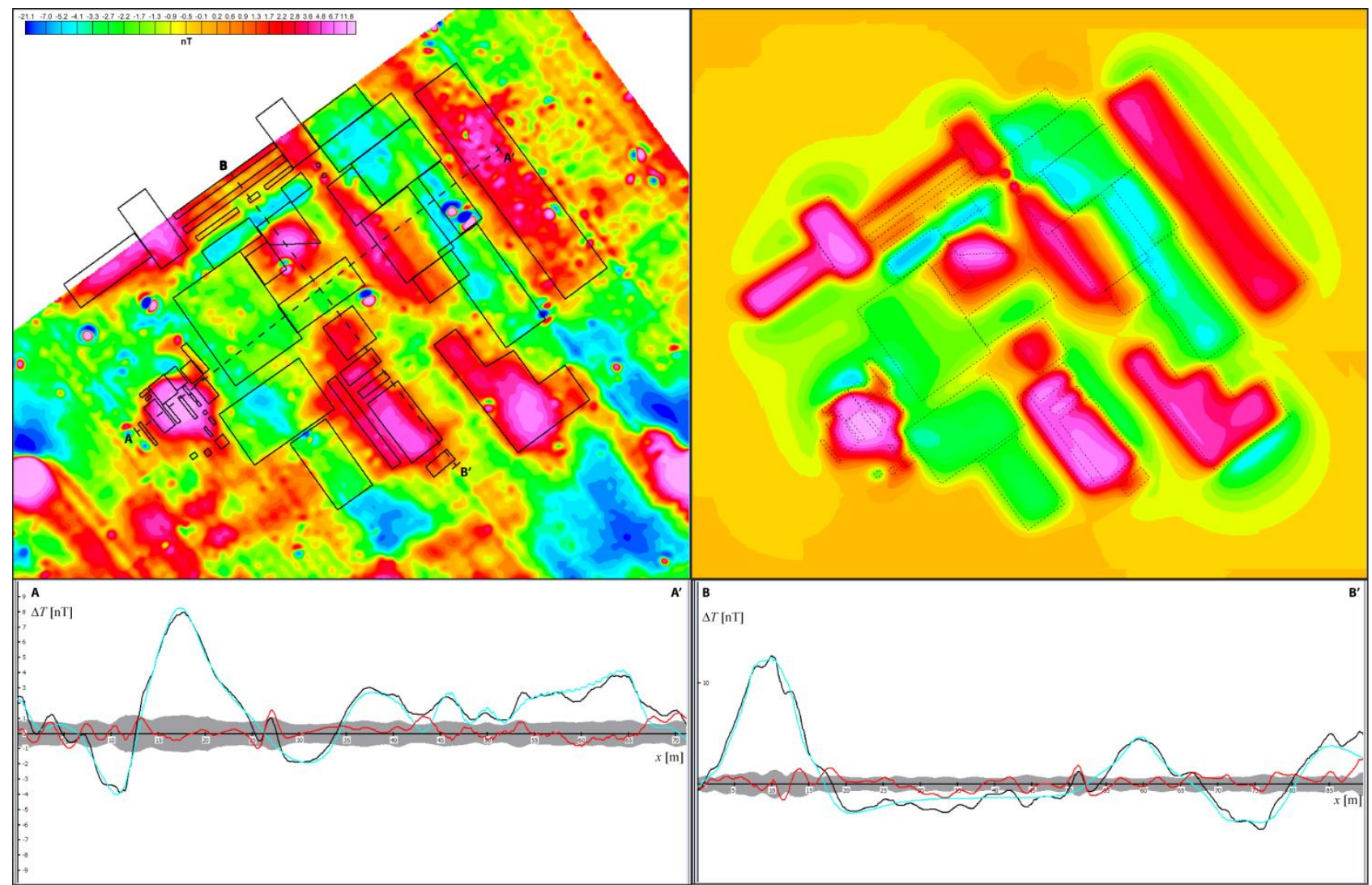

Figure 16. Modelling of area D of the northern sector of the Hadrianopolis settlement (see Fig. 10). Upper left panel shows the observed anomalies and a model of the underground that explains most of the observed signal in this rectangle (black lines). $\mathrm{A}-\mathrm{A}^{\prime}$ and $\mathrm{B}-\mathrm{B}^{\prime}$ are the traces of the two profiles shown in the upper left panels. Upper right panel shows a map of the theoretical anomalies, calculated from the block model. The lower panels illustrate the fit between observed and calculated anomalies along two profiles. 